\title{
Konditionale Konvergenz
}

Ideen, Institutionen und Standortwettbewerb in der Steuerpolitik von EU- und OECD-Ländern

Reformtrends in der Besteuerung von Kapitalgesellschaften und persönlichen Einkommen spielen in der Literatur über »Globalisierung « eine zentrale Rolle. Der offensichtlichste Trend ist die starke Abwärtsentwicklung der (Spitzen-)Steuersätze seit Mitte der achtziger Jahre. Der Beitrag analysiert die Konvergenzmuster bei Körperschaft- und Einkommensteuer und bewertet unterschiedliche Erklärungen der Abwärtstrends. Es wird gezeigt, dass bei beiden Steuersätzen konditionale Konvergenz sichtbar ist. Die Körperschaftsteuersätze haben sich zunehmend an der Ländergröße ausgerichtet, was auf die kausale Bedeutung des Steuerwettbewerbs schließen lässt. Die Spitzensteuersätze der persönlichen Einkommensteuer werden dagegen durch die Abgabenquote eines Landes beeinflusst, was auf das Fortbestehen nationaler Entscheidungsfreiheiten verweist. Die Analyse zeigt, dass sich die politischen Entscheidungsträger stärkeren materiellen Beschränkungen gegenüber sahen, als in der Literatur angenommen wird. Die kausale Bedeutung von Ideen muss entsprechend geringer eingeschätzt werden. Die Bedeutung der institutionellen Machtteilung in politischen Systemen variiert entsprechend der Stärke materieller Beschränkungen: Bei den Körperschaftsteuersätzen ist sie vernachlässigbar, bei den Spitzensteuersätzen erheblich.

\section{Einleitung ${ }^{1}$}

Reformtrends in der Besteuerung von Kapitalgesellschaften und persönlichen Einkommen spielen in der Literatur über die Auswirkungen der »Globalisierung « eine zentrale Rolle (Genschel 2003). Der offensichtlichste dieser Trends betrifft die nominalen (Spitzen-)Steuersätze - also die Sätze, die im Gesetz stehen und bei denen die Definition der steuerlichen Bemessungsgrundlage unberücksichtigt bleibt. Seit Ende der siebziger bzw. Mitte der achtziger Jahre sehen wir bei diesen Sätzen eine starke

1 Frühere Versionen dieses Papiers wurden im Politics and Economics-Seminar des Wissenschaftszentrums Berlin, im Workshop Sources of Cross-national Policy Convergence des Arbeitskreises »Politische Steuerung « der Deutschen Vereinigung für Politische Wissenschaft (DVPW) in Hamburg sowie im Rahmen des »Frankfurter Volkswirtschaftlichen Kolloquiums « der Universität Frankfurt präsentiert. Ich danke den Teilnehmern dieser Veranstaltungen, insbesondere Pablo Beramendi, Tom Cusack, Philipp Genschel, Achim Kemmerling und Alfons Weichenrieder, für konstruktive Kommentare und Diskussionen. Mein besonderer Dank gilt Matthias L. Maier, Thomas Plümper und den anonymen GutachterInnen der ZIB für hilfreiche Überarbeitungsvorschläge. Christian W. Martin danke ich für die Überlassung von Daten über Kapitalverkehrsbeschränkungen und Dennys Hill für Forschungsassistenz. Alle verbliebenen Unzulänglichkeiten gehen auf mein Konto. 
Abwärtsentwicklung. Besonders stark wurde der Trend nach den Steuerreformen Großbritanniens und der USA in den Jahren 1984 und 1986, denen eine Schock- und Katalysatorwirkung zugeschrieben wird (Messere 1997; Hallerberg/Basinger 1998). Zwischen 1983 und 2003 fielen Körperschaftsteuersatz und Spitzensteuersatz der persönlichen Einkommensteuer im Durchschnitt der Mitgliedsländer der Organisation für wirtschaftliche Zusammenarbeit und Entwicklung (OECD) um jeweils fast 20 Prozentpunkte: Ersterer von 49,5\% auf 32,4\%, Letzterer von $65,7 \%$ auf $47,6 \%{ }^{2}$ Dies entspricht bei den Körperschaftsteuersätzen einer Senkung von etwas über, bei den Spitzensteuersätzen von unter einem Drittel.

Die politikwissenschaftliche Literatur zu den Abwärtstrends diskutiert vor allem ihre Ursachen. Mit der Diskussion eng verkoppelt ist jedoch die Frage nach den möglichen Auswirkungen von Globalisierung und Steuerwettbewerb auf die Erreichbarkeit nationaler Politikziele, wie z. B. einer Konsolidierung des Haushalts oder der Umverteilung von Einkommen. Erkennt man nämlich die Ursachen der Abwärtstrends hauptsächlich im »endogenen « Wandel von policy-Ideen auf nationaler Ebene sowie in der internationalen Diffusion von Reformideen, so stellt sich die Frage nach der strukturellen Beschränkung nationalstaatlicher Politik durch offene Märkte nicht oder nur in stark abgeschwächter Form. Schreibt man dagegen den strukturellen Anreizen und Beschränkungen des Steuerwettbewerbs eine große Rolle bei der Erzeugung und Verstärkung der Abwärtstrends zu, dann stellt sich automatisch die Frage, wie gewichtig diese Beschränkungen aus nationaler Sicht tatsächlich sind. Im Folgenden stehen zunächst die Ursachen der Abwärtstrends im Mittelpunkt der Analyse. Abschließend werden jedoch die Implikationen der Analyse im Hinblick auf nationalstaatliche Entscheidungsfreiheiten in der Steuerpolitik ausführlich gewürdigt.

Was die Ursachen der Abwärtstrends angeht, stehen sich in der Literatur zwei Sichtweisen gegenüber. Einige Autoren sehen den Steuerwettbewerb als zentrale Triebkraft hinter den Steuersatzsenkungen. ${ }^{3}$ So schreibt etwa der renommierte Finanzwissenschaftler Richard A. Musgrave zusammenfassend:

»With increased involvement in trade and greater capital movement across national boundaries, progressive taxation, especially of capital income, becomes increasingly difficult to achieve at both the personal or corporate level. As the development of recent years has shown, the desire to attract capital or to prevent outflow leads to downward competition, uniformity, and the flattening of tax rates. [...] The built-in forces of globalization, rather than domestically held views of distributive justice, may well determine the future of progressive income taxation« (Musgrave 2002: 22f).

2 Die hier angegebenen Durchschnittswerte beziehen sich auf die gesamtstaatlichen Steuersätze, sie schließen also gliedstaatliche und lokale Steuern auf Einkommen ein. Streng genommen ist es falsch von »Körperschaftsteuern « zu sprechen, weil in einigen Ländern die lokalen Steuern keine Einkommensteuern im technischen Sinne sind (wie z. B. die deutsche Gewerbesteuer). Die Daten sind im Anhang erläutert. Die Werte sind ungewichtet und beziehen sich auf 21 OECD-Länder (»OECD«): Australien, Japan, Kanada, Neuseeland, Norwegen, die Schweiz und die USA sowie 14 Mitgliedsländer der Europäischen Union (EU) - Luxemburg und die zehn neuen Mitglieder werden nicht berücksichtigt.

3 Vgl. etwa Lee/McKenzie (1989); Hallerberg/Basinger (1998); Devereux et al. (2002). 
Für andere Autoren sind die Senkungen von Körperschaft- und Einkommensteuersätzen dagegen vor allem Ausdruck innerstaatlicher Lernprozesse und der internationalen Diffusion »neoliberaler « Ideen. ${ }^{4}$ Nationale Entscheidungsträger seien in ihrer Festlegung von Steuersätzen weiterhin relativ wenig eingeschränkt, nur hätten sich ihre Sichtweisen (Kognitionen) radikal verändert. So argumentiert etwa Geoffrey Garrett (1998b: 90), die Regierungen hätten schlicht aus Erfahrung gelernt und policy-Instrumente wie hohe Steuersätze als »ineffektiv« erkannt. Duane Swank und Sven Steinmo sprechen sogar von einem kognitiven »Paradigmenwechsel« (Swank/Steinmo 2002: 643; vgl. Hall 1993), im Zuge dessen sich nicht nur die Instrumente, sondern auch die Ziele der Regierungen massiv verschoben hätten von Gerechtigkeit zu Effizienz. Wie Steinmo zusammenfasst:

»It is tempting to argue [...] that the recent trend towards lower marginal tax rates for capital and high income earners witnessed around the world is a direct result of >globalisation $<$. But $[\ldots]$ these tax changes are also a product of the negative experience economists and policymakers had with high marginal tax rates in the 1970s to 1990s. [...] The bogey-man of >globalisation< and the threat of capital exit in the late 1980s and 1990s provided a powerful justification for tax policies which tax economists and tax policy advisors had long wanted to make - but [...] it is difficult to argue that it was international competition that >made us do it«« (Steinmo 2003: 229f).

Zwischen der stärker »materialistischen « und der stärker »ideenorientierten « Perspektive auf Einkommensteuerreformen in der OECD-Welt besteht kein grundsätzlicher Gegensatz. ${ }^{5}$ In beiden Ansätzen handeln individuelle Akteure aufgrund von Kognitionen (beliefs), in denen sich die materiellen oder strukturellen Beschränkungen bereits widerspiegeln (vgl. zu diesen Gemeinsamkeiten grundsätzlich Fearon/Wendt 2002; Maier 2003: 28-44). Freilich gibt es auch ideenorientierte Ansätze, die Ideen nicht als individuelle Kognitionen verstehen und die stärker an ihrer »konstitutiven « statt kausalen Rolle interessiert sind. Solche Ansätze stehen in einem stärkeren Gegensatz zu materialistischen Erklärungen (Brooks/Wohlforth 2000: 12, Fn. 15; Maier 2003: 44-62).

Die hier behandelten ideenorientierten Ansätze zur Steuerpolitik fallen jedoch nicht in diese Kategorie. Sie bemühen sich vielmehr darum, strukturelle Anreizstrukturen sowie deren Veränderungen zu modellieren und so ihren Einfluss auf den Wandel von Ideen zu präzisieren. Sie akzeptieren also den Punkt, den Stephen G. Brooks und William C. Wohlforth (2000: 6) in der Debatte über das Ende des Kalten Krieges gemacht haben: Ideenorientierte Erklärungen basieren auf einem impliziten oder expliziten Kontrast zu materialistischen Erklärungen. Welche Wahrnehmungen und Reformideen sich in politischen Prozessen durchsetzen, wird maßgeblich davon beeinflusst, wie gut diese Ideen mit den materiellen Beschrän-

4 Vgl. vor allem Garrett (1998b: 90); Swank (1998); Ottaviani (2002: 491f); Swank/ Steinmo (2002: 643); Steinmo (2003).

5 Der Begriff »materialistisch « wird hier in einem weiteren Sinne gebraucht. In Anlehnung an Judith Goldstein und Robert O. Keohane (1993) könnte man auch von »interessenorientierter « Erklärung sprechen. Gemeint sind Erklärungen, welche die strukturellen Beschränkungen betonen, denen sich die Akteure gegenübersehen. 
kungen in Einklang stehen. ${ }^{6}$ Um die Erklärungskraft von Ideen richtig einzuschätzen, braucht man deshalb möglichst gute materialistische Erklärungen als Kontrastfolie (vgl. auch Goldstein/Keohane 1993: 26-29). Der Konflikt zwischen Autoren wie Musgrave und Steinmo ist also kein methodischer, sondern ein substanzieller. Die eine Seite glaubt, dass die beobachtbaren Politiktrends durch sich ändernde materielle Bedingungen der globalen Ökonomie ausreichend erklärt werden können. Die andere Seite bestreitet dies und weist Ideen und ihrer internationalen Diffusion eine wichtigere kausale Rolle zu.

Das Ziel dieses Beitrags ist, zu einer angemessenen materialistischen Erklärung von Einkommensteuersätzen beizutragen, die nicht zuletzt als Kontrastfolie für ideenorientierte Erklärungen dienen kann. Ich werde zeigen, dass die Festlegung von Körperschaftsteuersätzen und Spitzensteuersätzen der Einkommensteuer strukturellen Beschränkungen unterliegt, die bisher nicht ausreichend berücksichtigt worden sind. Diese Beschränkungen resultieren teilweise tatsächlich aus dem internationalen Steuerwettbewerb. Insofern wird die Position von Musgrave und anderen gestützt. Zum Teil liegen die relevanten Beschränkungen jedoch auch im innerstaatlichen Bereich und wurden von beiden Seiten der Debatte vernachlässigt.

Um zu zeigen, dass strukturelle Beschränkungen tatsächlich bedeutsam waren, werde ich auf statistische Analysen der Steuersätze möglichst vieler vergleichbarer Länder zurückgreifen. Solche Analysen sind für die hier gewählte Fragestellung aus zwei Gründen besonders gut geeignet: Erstens machen Autoren wie Steinmo anders als viele andere Verfechter ideenorientierter Erklärungen - keine Aussagen über die Entscheidungsprozesse in einzelnen Ländern zu einzelnen Zeitpunkten. Es geht vielmehr um die Erklärung von Trends in der OECD-Welt insgesamt. Die empirische Analyse muss deshalb möglichst viele Länder in den Blick nehmen, was durch quantitative Analysen wesentlich leichter durchzuführen ist als durch Qualitative. Zweitens sind statistische Methoden zur Analyse struktureller Beschränkungen besonders gut geeignet, da Letztere unterschiedliche Länder unterschiedlich stark betreffen. Wenn die politischen Entscheidungsträger tatsächlich entsprechenden Beschränkungen unterliegen, so sollten sich beobachtbare Implikationen für den internationalen Vergleich von Steuersätzen ableiten und testen lassen.

In der quantitativen Analyse greife ich auf die Konzepte der ökonomischen Konvergenz-Literatur zurück (Sala-i-Martin 1996). Diese beschäftigt sich ebenfalls mit strukturellen Unterschieden zwischen Ländern, wenngleich von anderen Erkenntnisinteressen angeleitet. Unterliegen unterschiedliche Länder unterschiedlich starken Beschränkungen, so sollte es auch bei starkem Steuerwettbewerb nicht unbedingt zu einer Angleichung von Steuersätzen kommen. Stattdessen ist zu erwarten, dass sich die Steuersätze systematisch an der strukturellen Position der Länder innerhalb des Wettbewerbs ausrichten. Eine Konvergenz von Steuersätzen lässt sich nur dann fest-

6 Dies gilt unabhängig davon, ob bereits die Entwicklung von Ideen (Kognitionen) von materiellen Beschränkungen befördert worden war. Zwar wird Letzteres häufig der Fall sein, entscheidend ist hier jedoch nicht, woher Ideen kommen, sondern wie materielle Beschränkungen die Wahrscheinlichkeit beeinflussen, dass bestimmte Ideen in tatsächliche policies übersetzt werden; vgl. Brooks/Wohlforth (2002: 111). 
stellen, wenn man für die jeweiligen Strukturvariablen (im Folgenden: »Konditionierungsvariablen«) statistisch kontrolliert (Plümper/Schulze 1999: 451f). Man spricht in diesem Fall von »konditionaler Konvergenz«. Den Zusammenhang zwischen Konvergenztheorie und der Debatte um »Ideen « versus »Steuerwettbewerb « kann man stark vereinfacht folgendermaßen zusammenfassen: Die Art der Konditionierungszusammenhänge erlaubt Rückschlüsse darüber, wie wichtig der Steuerwettbewerb im Vergleich zu innerstaatlichen Beschränkungen war. Die Stärke von strukturellen Konditionierungszusammenhängen erlaubt Rückschlüsse darüber, wie wichtig die kausale Rolle von Ideen war.

Ich will im Folgenden zeigen, dass die beiden Arten von Grenzsteuersätzen Körperschaftsteuersatz und Spitzensatz der Einkommensteuer - für die hier betrachteten OECD- und EU-Länder ganz unterschiedliche Konvergenzmuster aufweisen. Für beide Steuersätze ist konditionale Konvergenz zu beobachten, jedoch sind die jeweils relevanten Konditionierungsvariablen ganz unterschiedlich. Die Körperschaftsteuersätze werden entscheidend durch die Ländergröße, gemessen an der Bevölkerungszahl, bestimmt. Dies steht im Einklang mit der theoretischen Literatur zum Steuerwettbewerb und zeigt, dass der Abwärtstrend bei der Körperschaftsteuer in großem Maße vom Steuerwettbewerb angetrieben wird. Die Spitzensteuersätze der persönlichen Einkommensteuer werden dagegen durch die Abgabenquote eines Landes beeinflusst, d. h. durch die Steuern und Abgaben in Prozent des Bruttoinlandsprodukts (BIP). Dieser Befund legt nahe, dass die Spitzensteuersätze keinem direkten Steuerwettbewerb ausgesetzt sind, sondern dass die Gesamtsteuerlast (des Faktors Arbeit) die Fähigkeit der Regierungen einschränkt, die Einkommensteuersätze abzusenken.

Ein weiterer, indirekter Test der hier vorgelegten Analyse struktureller Beschränkungen ist durch die Einbeziehung nationaler politischer Vetoinstitutionen möglich. Je stärker diese Beschränkungen sind, desto eher kann man erwarten, dass sich die policy-Präferenzen unterschiedlicher Vetospieler angleichen - gerade weil der strukturelle Druck auf die Kognitionen der Akteure stark ist (Ganghof 2003: 9-13). Wenn dies der Fall ist, dann ist auch bei einer hohen Anzahl von Vetopunkten policy-Wandel möglich (Tsebelis 2002: Kap. 2). Es ist deshalb zu erwarten, dass die Anzahl der Vetopunkte bei der Festlegung von Körperschaftsteuersätzen wesentlich weniger wichtig ist als bei der Festlegung von Spitzensteuersätzen. Letztere sind keinem direkten Steuerwettbewerb ausgesetzt und die Regierungen können die relevante Konditionierungsvariable, die Abgabenquote, zumindest mittelfristig selbst beeinflussen. Der strukturelle Druck in Richtung der Angleichung von policy-Präferenzen sollte somit geringer und die Rolle von Vetopunkten größer sein. Auch diese Hypothesen werden durch eine Regressionsanalyse unterstützt.

Die weitere Diskussion ist in vier Kapitel unterteilt. Das zweite Kapitel behandelt die relevante Theorie. Zunächst werden grundlegende konvergenztheoretische Unterscheidungen erläutert, dann werden die Probleme ideenorientierter Erklärungen für die Abwärtstrends der Körperschaft- und Spitzensteuersätze nacheinander diskutiert. Die anschließende empirische Analyse schreitet in zwei Schritten voran. Das dritte Kapitel untersucht, ob die absolute Streuung der Steuersätze zwischen 1983 und 2003 abgenommen hat, und analysiert die Bedeutung der beiden ange- 
sprochenen Konditionierungsvariablen - Ländergröße und Abgabenquote - in biund multivariaten Regressionen. Das vierte Kapitel testet formal auf konditionale Konvergenz, wobei der Rolle von Vetopunkten besondere Beachtung geschenkt wird. Das fünfte Kapitel fasst die Ergebnisse zusammen und behandelt darüber hinaus einige Auswirkungen des hier diagnostizierten Steuerwettbewerbs auf die Besteuerung von Kapitalgesellschaften.

\section{Theorie}

\subsection{Konvergenztheoretische Grundlagen}

In der konvergenztheoretischen Literatur werden zwei wichtige Unterscheidungen getroffen. Auf übergeordneter Ebene kann zwischen Beta-Konvergenz und SigmaKonvergenz unterschieden werden (vgl. Sala-i-Martin 1996: 1020-1022). Beta-Konvergenz bedeutet, dass Länder mit hohen Steuersätzen diese stärker senken. SigmaKonvergenz bedeutet, dass es zu einer Angleichung der Steuersätze kommt - dass also die Streuung der Steuersätze abnimmt. Beta-Konvergenz ist eine notwendige, aber keine hinreichende Bedingung für Sigma-Konvergenz. Wenn Länder mit höheren Steuersätzen stärkere Senkungen vornehmen, muss dies nicht unbedingt zu einer verringerten Varianz der Steuersätze führen. Es ist auch möglich, dass Länder nur die Plätze innerhalb der Verteilung tauschen. Anders formuliert: Bei Sigma-Konvergenz geht es darum, ob eine Verteilung im Zeitverlauf »schrumpft«, bei Beta-Konvergenz darum, wie »mobil« die Länder innerhalb einer gegebenen Verteilung sind.

Die zweite wichtige Unterscheidung ist die zwischen absoluter und konditionaler Beta-Konvergenz (vgl. Sala-i-Martin 1996: 1026-27; Plümper/Schulze 1999: 45152). Absolute Konvergenz von Steuersätzen wäre dann zu erwarten, wenn sich die Länder ausschließlich darin unterschieden, bei welchem Steuersatzniveau sie starten. Das Konzept konditionaler Konvergenz basiert dagegen auf der Annahme, dass sich Länder auch in anderen strukturellen Merkmalen unterscheiden, z. B. in der Ländergröße oder der Offenheit gegenüber dem Weltmarkt. Aus dieser Sicht ist nicht zu erwarten, dass alle Länder ihre Steuersätze auf ein einheitliches Niveau senken. Vielmehr sollten diejenigen Länder ihre Steuersätze stärker senken, die besonders weit von ihrer strukturell bestimmten »Gleichgewichtsposition « entfernt sind.

In der ökonomischen Literatur sind die »Konditionierungsvariablen« häufig lediglich Kontrollvariablen, die berücksichtigt werden müssen, bevor Beta-Konvergenz überhaupt festgestellt werden kann. Im Folgenden stehen die Konditionierungsvariablen jedoch im Mittelpunkt des Interesses, weil sie Rückschlüsse auf die Ursachen von Steuersenkungen zulassen. Ein zentrales Problem in der Diskussion über die Ursachen der Abwärtstrends liegt nämlich darin, dass beide Steuersätze Körperschaftsteuersatz und Spitzensatz der persönlichen Einkommensteuer - im Durchschnitt ganz ähnliche Abwärtsentwicklungen aufweisen, obwohl aus theoretischer Sicht der Wettbewerbsdruck auf die Körperschaftsteuersätze viel größer sein sollte als der auf die Spitzensteuersätze der persönlichen Einkommensteuer. Dies 
hat viele Autoren dazu geführt, für beide Arten von Steuersätzen ähnliche kausale Prozesse zu unterstellen. Die einen konstatieren einen Wettbewerbsdruck auf beide Steuersätze (Hallerberg/Basinger 1998; Wagschal 1999a, 1999b), die anderen bezweifeln die Relevanz solch eines Drucks für beide (Ottaviani 2002: 491f). Um diese entgegengesetzten Positionen zu bewerten, hilft die Analyse von Konvergenzmustern. Sie bestätigt die theoretische Erwartung, dass der Wettbewerbsdruck für die Körperschaftsteuersätze eine größere Bedeutung hat als für die Spitzensteuersätze der persönlichen Einkommensteuer.

Gleichzeitig zeigt die Analyse von Konvergenzmustern, dass die Entscheidungsträger bei der Festlegung von Einkommensteuersätzen wichtigen innerstaatlichen strukturellen Beschränkungen unterliegen, die von Verfechtern einer »ideenorientierten « Sichtweise vernachlässigt wurden. Die empirische Konvergenzanalyse qualifiziert somit die kausale Rolle von Reformideen für beide Arten von Steuersätzen. Bevor wir aber zur empirischen Analyse kommen, werden im Folgenden die theoretischen Grenzen ideenorientierter Erklärungen diskutiert. Dabei werden die Abwärtstrends bei Körperschaft- und Einkommensteuersätzen nacheinander behandelt.

\subsection{Körperschaftsteuersätze}

Am deutlichsten sind die Unterschiede zwischen einer »materialistischen « und einer »ideenorientierten « Sichtweise bei der Erklärung des Abwärtstrends bei den Körperschaftsteuersätzen. Die ideenorientierte Erklärung lässt sich folgendermaßen zusammenfassen: Die Regierungen hätten erkannt, dass die Kombination hoher Steuersätze mit einer Vielzahl von steuerlichen Spar- und Investitionsanreizen »ineffektiv« ist (Garrett 1998b: 90), weil sie unweigerlich zu stark unterschiedlichen Steuerbelastungen für unterschiedliche Typen von Kapitaleinkommen führt. Diese so genannten »intersektoralen« Steuersatzunterschiede (zwischen Kapitalgesellschaften und Personengesellschaften, einbehaltenen und ausgeschütteten Gewinnen etc.) greifen in Marktprozesse ein, was zwar früher gewünscht gewesen, aber zunehmend als kontraproduktiv erkannt worden sei (Steinmo 2003: 214-24). Um die Steuersatzdifferentiale zu reduzieren, hätten Regierungen die Steuersätze gesenkt und im Gegenzug die steuerliche Bemessungsgrundlage erweitert. Dies habe die Kapitalbesteuerung »marktkonformer « gemacht (Swank 1998). Der zugrunde liegende Lernprozess sei in großem Maße ein innerstaatlicher gewesen, aber die Reformen wichtiger Staaten, etwa der USA, hätten die nationalen Lernprozesse gestärkt und zu einer Diffusion »neoliberaler« Ideen geführt (Swank 2003).

Die ideenorientierte Erklärung beschreibt den Trend zu stärker marktkonformer Besteuerung treffend. Ihr Problem liegt jedoch darin, dass sie die allgemeine »Idee « marktkonformer - also nicht zwischen Sektoren unterscheidender - Besteuerung nicht hinreichend genau analysiert. Sie nimmt implizit an, dass es nur einen Pfad zu marktkonformer Besteuerung gegeben hat, welcher notwendigerweise mit Steuersatzsenkungen einherging. Dies ist jedoch falsch. Um dies zu verstehen, müssen wir einen kurzen Ausflug in die ökonomische Theorie unternehmen. 
Beim »alten« System hoher Steuersätze und spezifischer Spar- und Investitionsanreize ging es nicht nur darum, die Steuerlast zwischen Sektoren, sondern auch zwischen zwei analytischen Typen von Einkommen zu differenzieren: zum einen Kapitaleinkommen im engeren Sinne und zum anderen Reingewinne (vgl. Homburg 2000: 124-139, 329-340).

Kapitaleinkommen im engeren Sinne sind die Zahlungen, die notwendig sind, damit der Produktionsfaktor Kapital seine Leistung hervorbringt. Unternehmenseinkommen, die über diese reine »Entschädigung « der Produktionsfaktoren hinausgehen, nennt man ökonomische Renten. Ihre Besteuerung hat definitionsgemäß keinen Einfluss auf das Angebot an Produktionsfaktoren - zumindest in einer geschlossenen Volkswirtschaft. Eine wichtige Art von ökonomischer Rente ist der so genannte Reingewinn. Ihn kann man folgendermaßen verstehen: Wenn ein Investor eine Maschine kauft, dann braucht er dafür Geld. Mit diesem Geld hätte er aber auch eine sichere Finanzanlage tätigen können, die ihm einen bestimmten Zins eingebracht hätte. Der entgangene Zinsertrag muss durch den Kauf der Maschine zumindest wieder erwirtschaftet werden, damit die Investition überhaupt getätigt wird; die »Entschädigung « des Produktionsfaktors Kapital nennt man auch den Eigenkapitalzins. Er stellt das Kapitaleinkommen im engeren Sinne dar. Die Einkommen, die über die alternative Eigenkapitalverzinsung hinausgehen, nennt man dagegen Reingewinn.

Entscheidend ist nun Folgendes (vgl. Ganghof 2004: Kap. 2): Erstens spricht aus Sicht der Standard-Steuertheorie - in geschlossenen Volkswirtschaften - viel dafür, Reingewinne höher zu besteuern als normale Kapitaleinkommen, weil die Besteuerung von Reingewinnen für die Investitionen von bereits am Standort ansässigen Unternehmen weniger schädlich ist (z. B. Boadway/Bruce 1984). Zweitens diente das »alte« System hoher Steuersätze und spezifischer Investitionsvergünstigungen auch dazu, diese Verlagerung der Steuerlast auf Reingewinne zu erreichen. Wenn es nun einen Ideenwandel hin $\mathrm{zu} »$ marktkonformer« Besteuerung gegeben hat, dann ändert das nichts an der Rationalität höherer Steuersätze auf Reingewinne. Es wäre vielmehr zu erwarten gewesen, dass viele Regierungen auf Einkommensteuersysteme hinarbeiten, die zwar alle Sektoren des Kapitalmarkts gleichbehandeln, aber systematisch zwischen Reingewinnen und Kapitaleinkommen unterscheiden. So plädierte etwa der dänische Sachverständigenrat für Wirtschaftsfragen in seinem Frühjahrsgutachten 2001 sowohl für strikte Neutralität zwischen den Sektoren als auch für eine höhere Besteuerung von Reingewinnen: »Grundsätzlich sollten [...] normale Kapitaleinkommen und Reingewinne unterschiedlich behandelt werden. [...] Kapitaleinkommen sollten niedriger als derzeit besteuert werden, Reingewinne dagegen auf einem beträchtlich höheren Niveau « (Det Økonomiske Råd 2001: Kap. 2, eigene Übersetzung). Dementsprechend haben sich etwa die skandinavischen Länder und Italien um eine entsprechende Differenzierung der Steuerlast bemüht (vgl. Bordignon et al. 2001; Sørensen 2001). ${ }^{7}$

7 Die Extremform einer solchen Differenzierung liegt vor, wenn normale Kapitaleinkommen gar nicht besteuert werden. Man spricht dann von einer direkten Konsum- oder Ausgabensteuer. Sie wird auch in Deutschland seit langem von vielen Ökonomen gefordert. $\mathrm{Zu}$ den Details von Ausgabensteuern, ihrer »Position « im politischen Prozess und ihrer Beziehung zu anderen Reformmodellen siehe Ganghof (2004: Kap. 2, 5). 
Es gab noch einen zweiten Grund, hohe tarifliche Steuersätze (auf Reingewinne) beizubehalten. Die Körperschaftsteuer dient grundsätzlich als eine Art »Auffangbecken« (backstop) für die persönliche Einkommensteuer (Mintz 1995); sie soll vermeiden, dass die Bezieher hoher Einkommen Steuern umgehen können, indem sie eine Kapitalgesellschaft gründen. Aus dem selben Grund sollen Körperschaftsteuersatz und Spitzensatz der Einkommensteuer im Idealfall nicht zu stark auseinanderklaffen. Die Regierungen hätten deshalb einen guten Grund gehabt, die Körperschaftsteuersätze nicht so stark zu senken wie die Spitzensteuersätze der Einkommensteuer, um so die Lücke zwischen den beiden Sätzen zu schließen.

Die ideenorientierte Erklärung sinkender Körperschaftsteuersätze ist also theoretisch unvollständig. Ist die »materialistische « Steuerwettbewerbserklärung plausibler? Die Antwort ist positiv, weil hohe tarifliche Steuersätze und hohe Steuersätze auf Reingewinne einen erheblichen Wettbewerbsnachteil darstellen. Erstens veranlassen hohe nominale Steuersätze multinationale Unternehmen dazu, ihre Gewinne in Länder mit niedrigen Steuersätzen zu verlagern (Hallerberg/Basinger 1998; Ganghof 1999). Zweitens ist eine hohe steuerliche Belastung von Reingewinnen ein Standortnachteil für profitable Unternehmen, weil mit erhöhter Profitabilität der Anteil der Reingewinne an den Gesamtgewinnen zunimmt (Bond 2000). In dem Maße, in dem Länder um profitable Unternehmen konkurrieren, existiert also ein starker Anreiz, die Belastung der Reingewinne zu beschränken. Beide Probleme sind empirisch gut belegt (vgl. Devereux/Griffith 1998; Hines Jr. 1999; Devereux/Griffith 2003). Wenn deshalb die Körperschaftsteuersätze - auch die auf Reingewinne - auf breiter Front gesunken sind, so spricht dies für einen erheblichen Einfluss des Steuerwettbewerbs (vgl. Haufler/Schjelderup 2000; Devereux et al. 2002). Offensichtlich fühlten sich viele Regierungen »gezwungen«, die Körperschaftsteuersätze weiter abzusenken, als ihnen aus innerstaatlichen Gründen lieb war. In diesem Sinne setzt sich auch das obige Zitat des dänischen Sachverständigenrats fort:

»Es gibt jedoch verschiedene praktische Probleme bei der Einführung einer Körperschaftsteuer, die zwischen diesen beiden Arten von Gewinnen [normalen Kapitaleinkommen und Reingewinnen] unterscheidet. Insbesondere multinationale Unternehmen können häufig hohe tarifliche Körperschaftsteuersätze unterlaufen. Eine Art dies zu tun ist die Nutzung von Verrechnungspreisen, um Gewinne von Tochterfirmen in Länder mit niedrigen Körperschaftsteuersätzen zu verlagern « (Det Økonomiske Råd 2001: Kap. 2, eigene Übersetzung).

Nun können wir den Einwand gegen die ideenorientierte Erklärung klar benennen. Diese Erklärung ist theoretisch unvollständig, weil sie nicht alle relevanten Reformmodelle erfasst. Dadurch wird es unmöglich, alternative Reformpfade zu spezifizieren und den Einfluss materieller Beschränkungen angemessen zu verstehen. Letztlich leidet sie unter einer Form von selection bias (Auswahlverzerrung), weil sie sich nur mit den spezifischen Reformideen beschäftigt, die im politischen Prozess tatsächlich erfolgreich waren. Führt man eine umfassende Analyse von theoretisch vorhandenen und tatsächlich diskutierten Reformpfaden durch, so zeigt sich, dass der pauschale Hinweis auf »policy-Lernen« oder einen Ideenwandel die Senkung von nominalen Körperschaftsteuersätzen nicht erklären kann. Vielmehr drängt sich die 
Vermutung auf, dass ein zunehmend starker Steuerwettbewerb den politischen Entscheidungsträgern auf nationaler Ebene wenig Wahlfreiheit gelassen hat.

Um diese Hypothese quantitativ zu testen, können wir auf ein robustes Resultat der ökonomischen Theorie des Steuerwettbewerbs zurückgreifen, wonach die Konvergenz von Kapitalsteuersätzen von der Bevölkerungsgröße eines Landes konditioniert werden sollte. ${ }^{8}$ Senkt ein kleines Land den Steuersatz auf Kapital, so gibt es aufgrund des kleinen existierenden Kapitalstocks wenig an Steuereinnahmen zu verlieren. Gleichzeitig sind durch die Steuersatzsenkung jedoch relativ hohe Steuereinnahmen aus Kapitalzuflüssen zu erwarten. Gemessen wird die Ländergröße als Logarithmus der Bevölkerungszahl (lnpop) (vgl. Fuest/Weichenrieder 2002; Slemrod 2004; siehe Anhang).

\subsection{Spitzensteuersätze der persönlichen Einkommensteuer}

Die ideenorientierte Erklärung für den Abwärtstrend bei den Spitzensteuersätzen der persönlichen Einkommensteuer ist ganz ähnlich wie für den Trend bei den Körperschaftsteuersätzen, allerdings theoretisch noch unspezifizierter und unvollständiger. Es wird angenommen, dass die neue Logik von »Steuersatzsenkung plus Verbreiterung der Bemessungsgrundlage« auch für die persönliche Einkommensteuer gilt (Steinmo 2003: 215-223). Swank und Steinmo (2002: 643) argumentieren sogar, dass der Ideenwandel in der Einkommensteuerpolitik mit dem Konzept des »Paradigmenwechsels« beschrieben werden kann, das Peter Hall (1993) am Beispiel des Wechsels vom Keynesianismus zum Monetarismus in der makroökonomischen Politik entwickelt hat. Es hätten sich nicht nur die Instrumente, sondern auch die Ziele der Steuerpolitik verändert: von Gerechtigkeit hin zu Effizienz. Unter dem Eindruck vergangener Misserfolge hätten sich die Kognitionen linker und rechter Politiker stark angeglichen. Überall hätten Reformer auf eine starke Abflachung des Steuertarifs gesetzt. Wie Steinmo ausführt:

»The convergence of views has been remarkable indeed. [...] In the last eight years, this author interviewed ministry of finance officials in seven OECD countries from Australia and Japan to Sweden and Denmark. In every single case, I have heard remarkably similar beliefs. This view is best summarized in the following statement made by the former Swedish Minister of Finance, Kjell Olof Feldt: > The very high level of progressive taxation just does not work « (Steinmo 2003: 221, Hervorh. dort).

Bei genauerer Betrachtung ist auch diese ideenorientierte Sichtweise unvollständig. Ihr analytischer Kern liegt - wie schon bei den Körperschaftsteuersätzen - in den negativen Erfahrungen, welche die politischen Eliten mit den stark unterschiedlichen Steuerbelastungen auf unterschiedliche Arten von Kapitaleinkommen gemacht haben (Steinmo 2003: 219). Die steuerlich stark privilegierten Arten von Kapitaleinkommen konnten von Steuerzahlern mit hohen Einkommen als »Steuerschlupflöcher« missbraucht werden. Ein Weg, diese Schlupflöcher zu schließen, liegt in der 
Tat darin, die Steuersätze auf Kapital- und Arbeitseinkommen generell abzuflachen und anzugleichen. Dieses Konzept einer »umfassenden«, aber stark abgeflachten Einkommensteuer erfreut sich auch in Deutschland großer Beliebtheit. Es gibt jedoch ebenso einen anderen nahe liegenden Weg: die stärkere Trennung von Kapital- und Arbeitsbesteuerung. Durch eine solche Trennung fällt es leichter, die Kapitalsteuersätze zu senken und anzugleichen und so Schlupflöcher zu schließen; gleichzeitig reduziert sich die Notwendigkeit, die Progression in der Arbeitsbesteuerung abzusenken.

Ein Beispiel: In den nordischen Ländern konnten die Eigentümer selbst genutzten Wohneigentums ihre Schuldzinsen steuerlich geltend machen - bis zu Spitzensteuersätzen von zeitweise 88 Prozent (Schweden). Die impliziten Mieteinnahmen, die aus dieser Investition ins Eigenheim entstanden, wurden jedoch nicht oder kaum besteuert. Der »Sektor« selbst genutzten Wohneigentums eröffnete somit ein Steuerschlupfloch. Steuerzahler mit hohen Einkommen hatten einen Anreiz, sich zu verschulden, um somit ihre Steuerlast zu drücken. Die Resultate waren

- große Einnahmeverluste des Staates in der Kapitalbesteuerung, die höhere Steu-

ersätze auf Arbeitseinkommen notwendig machten;

- ineffiziente Investitionsmuster;

- eine hohe Verschuldung der privaten Haushalte;

- eine reduzierte effektive Progressivität der Einkommensteuer.

Die Regierungen in den entsprechenden Ländern reagierten darauf in folgender Weise: Sie legten einen einheitlichen, niedrigen und proportionalen Steuersatz auf Kapitaleinkommen fest, der zwischen 25 und 30 Prozent lag und es ihnen leichter machte, die Erträge aus selbst genutztem Wohneigentum in die Besteuerung einzubeziehen. Statt jedoch den Spitzensteuersatz auf Arbeitseinkommen auch auf dieses Niveau abzusenken, wurde die Besteuerung von Arbeits- und Kapitaleinkommen stärker getrennt. Ein Haupteffekt der Trennung war, dass sich die nordischen Staaten nicht mehr bis zu den ebenfalls abgesenkten Spitzensteuersätzen auf Arbeit, die nun zwischen 55 und 70 Prozent lagen, an den Zinskosten der Haushalte beteiligten, sondern nur bis zum viel niedrigeren Kapitalsteuersatz. Dadurch wurden Steuerschlupflöcher geschlossen und die Steuereinnahmen erhöht. Gleichzeitig konnte eine progressive Besteuerung der Arbeitseinkommen mit relativ hohen Spitzensteuersätzen erhalten bleiben.

Der vermeintliche »Paradigmenwechsel « hin zu einer marktkonformen Kapitalbesteuerung mit niedriger und gleichmäßiger Steuerbelastung auf unterschiedliche Typen von Kapitaleinkommen impliziert also nicht automatisch stark abgesenkte Spitzensteuersätze auf Arbeit. Wie bei den Körperschaftsteuersätzen gibt es nicht nur eine Antwort auf die negativen Erfahrungen der Vergangenheit, sondern mindestens zwei:

- die Absenkung aller Steuersätze auf ein einheitliches, niedriges Niveau (»umfassende« oder »synthetische« Einkommensteuer);

- die systematische Trennung von Kapital- und Arbeitsbesteuerung (»duale « Einkommensteuer). 
Natürlich sprachen aus dem Blickwinkel der Reformer gute Gründe für die erste Option. Eine Trennung von Kapital- und Arbeitsbesteuerung ist administrativ nicht immer einfach ausführbar und kann zu neuen Steuerschlupflöchern führen. Gleichzeitig gibt es jedoch auch starke strukturelle Anreize für die Beibehaltung relativ hoher Spitzensteuersätze auf Arbeitseinkommen (vgl. zu den folgenden Ausführungen Ganghof 2004: Kap. 4). Vereinfacht gesagt: Je höher die gesamte Steuer- und Abgabenlast, desto höher ist die Belastung des Faktors Arbeit; je höher die Belastung des Faktors Arbeit, desto höher muss der Spitzensteuersatz sein, um ein gewisses $\mathrm{Ma}$ an Progressivität in der Arbeitsbesteuerung zu erreichen. Die Freiheit der Regierungen, zwischen den beiden Reformmodellen (oder -ideen) zu wählen, ist also wiederum strukturell beschränkt. In Hochsteuerländern ist die Wahrscheinlichkeit, dass sich Ideen einer umfassenden, aber stark abgeflachten Einkommensteuer durchsetzen, niedriger als in Niedrigsteuerländern.

Bei genauerer Betrachtung müssen zwei Fälle von Hochsteuerländern unterschieden werden. In einigen Ländern, z. B. in Dänemark oder Schweden, geht eine hohe Gesamtsteuerlast mit einer hohen Einkommensteuerlast einher ( $>20 \%$ des BIP). In diesen Ländern würde deshalb der Versuch, eine umfassende Einkommensteuer mit niedrigen Steuersätzen einzuführen, $\mathrm{zu}$ erheblichen Steuerausfällen führen. In Dänemark etwa gibt es heute immer noch einen Spitzensteuersatz von ca. 60 Prozent, der bereits unterhalb des Lohns eines durchschnittlichen Industriearbeiters einsetzt. Der Versuch, den Spitzensteuersatz stark abzusenken, z. B. auf den Körperschaftsteuersatz von 30 Prozent, würde unweigerlich zu massiven Einnahmeverlusten führen. In anderen Hochsteuerländern wie Frankreich, Deutschland oder den Niederlanden gibt es traditionell relativ »kleine « Einkommensteuern $(<15 \%$ des BIP), weil der Sozialstaat in großem Maße über Sozialabgaben und Mehrwertsteuern finanziert wird (vgl. Ganghof 2000; Wagschal 2001). Dies würde es den Regierungen einerseits eher erlauben, die Einkommensteuer stark abzuflachen, ohne Einnahmen zu verlieren. Andererseits ist dies aber eben nur deshalb der Fall, weil der Sozialstaat weitgehend über »Steuern« finanziert wird, die in großem Maße proportionale oder regressive Steuern auf den Faktor Arbeit darstellen. Es gibt deshalb in diesen Ländern einen strukturellen Anreiz, die Einkommensteuer relativ progressiv zu gestalten. Die Einkommensteuer erfüllt also teilweise die Funktion der »Progressivitätsanpassung«, wodurch die Fähigkeit bzw. der Wille der Regierungen, den Einkommensteuertarif radikal abzuflachen, beschränkt wird.

Fassen wir zusammen: Steinmo und andere mögen Recht haben, wenn sie betonen, dass sich Reformer verschiedener Parteien in praktisch allen OECDLändern auf ähnliche Reformprinzipien berufen haben. Gleichzeitig unterlagen diese Politiker jedoch bei der Umsetzung dieser Ideen in tatsächliche Politik ganz unterschiedlichen, strukturellen Beschränkungen. Diese müssen berücksichtigt werden, bevor die kausale Rolle von Ideen angemessen eingeschätzt werden kann. Ich habe argumentiert, dass die Festlegung von Spitzensteuersätzen grundlegend durch die Steuer- und Abgabenquote eines Landes beschränkt wird, d. h. durch die Steuern und Abgaben in Prozent des Bruttoinlandsprodukts. Diese Hypothese wird im folgenden Abschnitt getestet, wobei - wie üblich - die Abgabenquoten der OECD Rev- 
enue Statistics Verwendung finden (taxtotal). ${ }^{9}$ Im vierten Abschnitt werden auch die Beitrittsländer der EU einbezogen; für einige dieser Länder stammen die Abgabenquoten aus anderen Quellen (vgl. Anhang).

\section{Sigma-Konvergenz und Konditionierungsvariablen}

Der dritte Abschnitt beginnt die empirische Analyse und verfolgt zwei Ziele: Erstens wird eine einfache Analyse der Sigma-Konvergenz durchgeführt, indem die Entwicklung der Standardabweichung im Zeitverlauf verfolgt wird. Es wird also überprüft, ob die absolute Streuung der Steuersatzverteilung abgenommen hat. Die Standardabweichung enthält jedoch keine Information über die Position einzelner Länder innerhalb der Steuersatzverteilung. Das zweite Ziel ist es zu zeigen, dass Ländergröße und Abgabenquote tatsächlich wichtige Konditionierungsvariablen für Körperschaft- bzw. Spitzensteuersätze (geworden) sind. ${ }^{10}$ Ich beginne mit einer uniund bivariaten Analyse über die gesamte Periode 1983-2003 und teste anschließend die Robustheit der Ergebnisse anhand von multivariaten Regressionen für das Jahr 2003. ${ }^{11}$

\subsection{Uni- und bivariate Analyse}

Tabelle 1 (siehe Seite 20) bildet die Konvergenzmuster für die Periode 1983-2003 in Abständen von jeweils fünf Jahren ab. Für beide Steuersätze werden jeweils fünf Variablen dargestellt. Die erste Variable ist die Standardabweichung als einfache Maßzahl für die absolute Streuung der Steuersätze. Nimmt die Standardabweichung im Zeitverlauf ab, hat Sigma-Konvergenz stattgefunden. Des Weiteren sind die niedrigsten und höchsten Werte der jeweiligen Steuersatzverteilung dargestellt. Sie geben einen Hinweis darauf, in welchem Abschnitt der Verteilung die stärksten Senkungen stattgefunden haben. Die letzten beiden Variablen beziehen sich auf bivariate Regressionen des jeweiligen Steuersatzes (corprate und topprate) auf die dazugehörige Konditionierungsvariable (lnpop und taxtotal). Es wird zum einen der Regressionskoeffizient für die Konditionierungsvariable dargestellt, zum anderen $\mathrm{R}^{2}$ als Maßzahl dafür, wie viel der Streuung in den Steuersätzen durch die Konditionierungsvariable »erklärt « wird. In den Regressionen für den Spitzensteuersatz (topprate) wird Japan als Ausreißer vernachlässigt. Japan hat während der gesamten Pe-

9 Diese Quoten sind aufgrund ihrer Verfügbarkeit für längere Zeiträume fast alternativlos, jedoch nicht völlig unproblematisch; vgl. die Diskussion in Ganghof (2004: Kap. 4).

10 Es ist zu beachten, dass die beiden unterschiedlichen Konditionierungsvariablen für die hier betrachteten Ländergruppen keineswegs so hoch korreliert sind, wie man dies vielleicht vermuten könnte. Im Jahr 2001 betrug Pearsons $r$ für die hier primär betrachtete Gruppe von 21 OECD-Ländern (»OECD«) -0,38. Für die Vereinigungsmenge dieser Länder und der Mitgliedsstaaten der erweiterten EU (»OECD/EU«) betrug der Korrelationskoeffizient nur $-0,08$.

11 Alle Analysen wurden mit STATA 8.0 erstellt. 
riode eine äußerst niedrige Abgabenquote, aber - trotz starker Senkungen - einen hohen Spitzensteuersatz. In der multivariaten Analyse des nächsten Abschnitts wird Japan einbezogen und verliert seinen Ausreißer-Status.

Tabelle 1: $\quad$ Sigma-Konvergenz und bivariate Analyse der Konditionierungsvariablen (»OECD«)

\begin{tabular}{|c|c|c|c|c|c|c|c|c|c|c|}
\hline \multirow{2}{*}{$\frac{\text { Variable }}{\text { Indikator }}$} & \multicolumn{5}{|c|}{ Körperschaftsteuersatz (corprate) } & \multicolumn{5}{|c|}{ Spitzensteuersatz (topprate) } \\
\hline & $\mathbf{S A}$ & $\min$ & $\max$ & Beta & $\mathbf{R}^{2}$ & $\mathbf{S A}$ & $\min$ & $\max$ & Beta & $\mathbf{R}^{2}$ \\
\hline 005 & 7,8 & 33,0 & 0,0 & 0,50 & $1 \%$ & 11,2 & 44,2 & & 0,8 & $40 \%$ \\
\hline 88 & 8,9 & 28,0 & 62 & 0,28 & $0 \%$ & 13,2 & 31 & 7 & 1,2 & 54 \\
\hline 1993 & 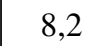 & 25,0 & 5 & 3,7 & 33 & 9,4 & 3 & & 0,9 & 48 \\
\hline 08 & 2 & 27,8 & 56,4 & 3,6 & $41 \%$ & 8,4 & 33,0 & 65,0 & 0,6 & $32 \%$ \\
\hline 2003 & 6,3 & 12,5 & 41,8 & $3,48 * * *$ & $47 \%$ & 6,2 & 38,6 & 59,0 & $0,76^{* * *}$ & $68 \%$ \\
\hline
\end{tabular}

Anmerkungen: Die Berechnungen für corprate beziehen sich auf 21 OECD-Länder (,,OECD“). Bei den Berechnungen für topprate wird Japan vernachlässigt (vgl. Text). SA = Standardabweichung; $\boldsymbol{m i n}=$ niedrigster Wert $; \boldsymbol{m a x}=$ höchster Wert $;$ Beta $=$ Beta-Koeffizient der Kleinste-Quadrate-Regression des Steuersatzes (corprate bzw. topprate) auf seine jeweilige Konditionierungsvariable (lnpop bzw. taxtotal); Sternchen stehen für das Signifikanzniveau: $* * *=p<0,01 ; \boldsymbol{R}^{2}=$ erklärte Varianz der bivariaten Regression in Prozent.

Die Entwicklung der Standardabweichung im Zeitverlauf zeigt, dass Sigma-Konvergenz stattgefunden hat, bei den Spitzensteuersätzen deutlich stärker als bei den Körperschaftsteuersätzen. Diese Konvergenz wird noch deutlicher, wenn man sich auf die Periode ab 1988 konzentriert. Im Zuge der Steuerreformen in den VorreiterStaaten, insbesondere in Großbritannien 1984 und in den USA 1986, hat sich die Streuung der Steuersätze zunächst vergrößert. Erst nachdem die Auswirkungen dieser Reformen besser absehbar waren, gab es eine stetige Verringerung der Standardabweichung - von 8,9 auf 6,3 für die Körperschaftsteuersätze und von 13,2 auf 6,2 für die Spitzensteuersätze der persönlichen Einkommensteuer. Die Streuung der Spitzensteuersätze war also 1983 deutlich höher als die der Körperschaftsteuersätze und hat sich im Lauf der Zeit an Letztere angepasst. Dies ist vor allem durch die Senkung besonders hoher Steuersätze erreicht worden. Bei der Körperschaftsteuer sind die Werte der Minima und Maxima in etwa gleichem Maße gesunken, jeweils um ca. 20 Prozentpunkte; bei der Einkommensteuer ist der höchste Wert dagegen viel stärker gesunken als der niedrigste, 34 stehen hier lediglich 5,6 Prozentpunkten gegenüber.

Was die Ergebnisse der bivariaten Regressionen betrifft, so gab es in den achtziger Jahren keinen Zusammenhang zwischen Ländergröße und Körperschaftsteuersatz. Im Zuge des Abwärtstrends nach der US-Reform von 1986 entstand jedoch solch ein Zusammenhang. Für 1993, 1998 und 2003 ergeben sich Regressionskoeffizienten von einheitlicher Größe und hoher statistischer Signifikanz. Der durch die Ländergröße »erklärte« Teil der Varianz in den Steuersätzen steigt im Zeitverlauf an und erreicht 2003 fast 50\%. Ein ähnliches Muster zeigt sich für den Zusammenhang 
zwischen Abgabenquote und Spitzensteuersatz, allerdings mit dem wichtigen Unterschied, dass Letzterer bereits im Jahr 1983 statistisch signifikant war und trotz des Abwärtstrends nicht an »Erklärungskraft« verloren hat. Diese Ergebnisse legen einerseits nahe, dass der Steuerwettbewerb in der Tat eine wichtige Triebkraft hinter den Senkungen der Körperschaftsteuersätze war - anders ist die zunehmende Ausrichtung dieser Sätze an der Ländergröße kaum zu erklären. Andererseits wird deutlich, dass die Absenkung von Spitzensteuersätzen durch die Gesamtsteuerlast beschränkt wurde. Dadurch war es insbesondere kleinen Hochsteuerländern praktisch unmöglich, die beiden Steuersätze auf ein einheitliches Niveau abzusenken.

Diese Interpretationen werden durch vergleichende Fallstudien gestützt (Ganghof/ Eccleston 2004; Ganghof 2005). Zunächst zur Körperschaftsteuer: Die Entscheidungsträger in den OECD-Regierungen waren sich über die Mechanismen des Steuerwettbewerbs im Klaren und sahen die dringende Notwendigkeit, den Körperschaftsteuersatz an »internationale Standards« anzupassen. Insbesondere die Sequenz von Entscheidungen in vielen Ländern verdeutlicht die große Bedeutung des Steuerwettbewerbs bei der Körperschaftsteuer und seine viel geringere Bedeutung bei der persönlichen Einkommensteuer. Zum Beispiel haben Australien, Neuseeland und Dänemark ihre Körperschaftsteuersätze direkt vor der US-Reform von 1986 erhöht, um sie am Spitzensteuersatz für persönliche Kapitaleinkommen auszurichten; in Deutschland gab es eine entsprechende Angleichung der Sätze bereits. Im Zuge des verschärften Steuerwettbewerbs mussten jedoch alle vier Länder den Körperschaftsteuersatz deutlich senken und einen mehr oder weniger großen Abstand zum Spitzensteuersatz der Einkommensteuer akzeptieren. In Australien überlebte das System der angeglichenen Steuersätze nur zwei Jahre.

Weitere Einsichten liefern Detailanalysen der nationalen policy-Diskurse über größere Zeiträume hinweg (Ganghof 2004, 2005). Es gab zahlreiche Regierungen, die grundsätzlich gern an hohen Körperschaftsteuersätzen und selektiven Investitionsanreizen festgehalten hätten, dies aber angesichts des Steuerwettbewerbs nicht konnten. Zu diesen Ländern gehören Australien, Dänemark, Kanada, aber auch Deutschland, wo sich insbesondere die SPD als Regierungspartei gezwungen sah, die Abschreibungsbedingungen deutlich stärker als angekündigt zu verschlechtern, um so die notwendigen Steuersatzsenkungen zu finanzieren. In anderen Ländern wie Österreich, Großbritannien, Kroatien oder Italien wurde darüber diskutiert, die Unternehmensteuerlast noch stärker und systematischer auf Reingewinne zu verlagern (vgl. Kapitel 2); der Steuerwettbewerb war einer der Gründe dafür, warum diese Reformansätze gar nicht verfolgt, abgeschwächt oder wieder abgeschafft wurden (Isaac 1997; Wala 1999; Bordignon et al. 2001; Keen/King 2003). Kurz: Es war der Steuerwettbewerb, der dazu geführt hat, dass die Steuersatz-Präferenzen von Regierungen unterschiedlicher Couleur bei der Körperschaftsteuer konvergierten.

Bei der persönlichen Einkommensteuer war die Situation anders: Hier gab es keinen oder nur einen sehr schwachen Wettbewerbsdruck, weil Arbeitnehmer weit weniger mobil sind als die Gewinne von Kapitalgesellschaften. Die Absenkung absurd hoher Spitzensteuersätze von bis zu 93\% - dieser Wert wurde in Japan erreicht - kann mit policy-Lernen gut erklärt werden und musste auch keineswegs 
zu Einnahmeverlusten oder einer niedrigeren effektiven Progression führen, weil im Gegenzug Steuerschlupflöcher geschlossen wurden. Teilweise wurden diese Schlupflöcher gerade dadurch geschlossen, dass Arbeits- und Kapitalbesteuerung innerhalb der Einkommensteuer systematischer voneinander getrennt wurden (vgl. Kapital 2). Gleichzeitig hat die Trennung von Arbeits- und Kapitalbesteuerung den Entscheidungsträgern die Freiheit gegeben, die progressive Einkommensteuer auf Arbeitseinkommen weiter als Instrument der »Progressivitätsanpassung « innerhalb des Gesamtsystems der Lohnbesteuerung einzusetzen. Aus diesem Grund ist der Zusammenhang zwischen Abgabenquote und Spitzensteuersatz bestehen geblieben. Hätten die Regierungen - insbesondere in Hochsteuerländern - versucht, ihren Spitzensteuersatz auf dasselbe Niveau wie den Körperschaftsteuersatz zu senken, hätte dies zu großen Einnahmeverlusten und/oder einer erheblich verminderten Progression in der Lohnbesteuerung geführt. Ein kleines Hochsteuerland wie Schweden ist deshalb bereit, einen Spitzensteuersatz zu akzeptieren, der doppelt so hoch ist wie der Körperschaftsteuersatz - Ersterer liegt bei 56 Prozent, Letzterer bei 28 Prozent.

\subsection{Multivariate Analyse}

Die bisherigen Analysen waren bewusst einfach gehalten, da die Daten eine relativ deutliche Sprache sprechen. Nun soll untersucht werden, wie robust die obigen Resultate sind, wenn man weitere Kontrollvariablen hinzufügt, weitere Länder in die Analyse einbezieht und den japanischen Ausreißer wieder in die Analyse der Spitzensteuersätze einbezieht. Dazu führe ich multivariate Regressionen für die Steuersätze im Jahr 2003 für drei verschiedene Ländergruppen durch:

- für die bisher betrachteten $21 \mathrm{OECD}$-Länder (»OECD«);

- für die Vereinigungsmenge dieser Gruppe und der erweiterten EU (»OECD/ $\mathrm{EU} \ll)$

- für alle Länder aus dem »corporate tax survey« der Wirtschaftsprüfungsgesellschaft KPMG, bei denen Daten für die unabhängigen Variablen verfügbar waren $(\gg \mathrm{KPMG} \ll)$.

Welche Kontrollvariablen müssen berücksichtigt werden? Diese Frage ist nicht leicht zu beantworten, da weder die theoretische Literatur noch frühere empirische Analysen viel Erfolg dabei hatten, das Niveau von (Spitzen-)Steuersätzen im internationalen Vergleich zu erklären (vgl. Garrett 1998a; Slemrod 2004). Vor allem drei Variablen erscheinen bedeutsam (vgl. Anhang):

Erstens erwarten einige Theoretiker einen Zusammenhang zwischen Körperschaftsteuersatz und Spitzensteuersatz der Einkommensteuer, weil die Körperschaftsteuer zum Teil als »Auffangbecken « (backstop) der persönlichen Einkommensteuer konzipiert ist (Mintz 1995; Slemrod 2004). Dies ist der Grund, weshalb Steuerexperten die Spreizung zwischen den beiden Sätzen gering halten wollen. Die folgenden Regressionen kontrollieren deshalb jeweils für den (Spitzen-)Steuersatz des anderen Sektors (corprate bzw. topprate). Damit erübrigt sich gleichzeitig die Notwendigkeit, die Konditionierungsvariablen des jeweils anderen Sektors einzube- 
ziehen. Würde man etwa Körperschaftsteuersatz und Ländergröße gleichzeitig zur »Erklärung« der Spitzensteuersätze heranziehen, käme es zu einem Endogenitätsproblem. Ich konzentriere mich im Folgenden insofern auf die kausal nähere Variable (vgl. King 1991: 1050); sollte die Ländergröße einen Einfluss auf den Spitzensteuersatz der persönlichen Einkommensteuer haben, so wäre dieser Effekt zu einem großen Teil durch den Körperschaftsteuersatz vermittelt. Analoges gilt für die Abgabenquote.

Zweitens ist es möglich, dass Länder mit geringerer Wirtschaftskraft niedrigere Steuersätze wählen, um ihr Wachstum zu beschleunigen. So findet etwa Harry Grubert (2001) für eine Auswahl von 50 Ländern, dass in ärmeren Ländern die durchschnittliche effektive Steuerlast für US-Firmen zwischen 1984 und 1992 stärker gefallen ist als in reicheren Ländern. ${ }^{12}$ Wie Grubert verwende ich das Prof-KopfBIP eines Landes als Indikator (gdppc).

Drittens könnte man vermuten, dass sich Länder mit stärkeren Kapitalverkehrsbeschränkungen höhere Körperschaftsteuersätze leisten können. Allerdings ist dabei zu beachten, dass die hier vorrangig interessierende Ländergruppe (»OECD«) spätestens seit Mitte der neunziger Jahre weitgehend frei von Kapitalverkehrskontrollen war, sodass es für die nachfolgende Periode kaum Varianz gibt, die zur Erklärung der Steuersätze herangezogen werden könnte. Kapitalverkehrskontrollen sollten deshalb - wenn überhaupt - in größeren Samples eine Rolle spielen. Ich verwende daher für das »KPMG «-Sample einen Indikator für die Beschränkungen der Direktinvestitionen (longcap1). ${ }^{13}$

Tabelle 2 (siehe folgende Seite) stellt die Regressionsergebnisse dar. Alle Regressionen enthalten jeweils die Konditionierungsvariable (Inpop bzw. taxtotal), den Steuersatz des jeweils anderen Sektors (topprate bzw. corprate) sowie die Wirtschaftskraft (gdppc) als exogene Variablen. Die Regression für »KPMG« kontrolliert zusätzlich für Kapitalverkehrskontrollen. Das entscheidende Ergebnis sei vorweg betont: Die Koeffizienten beider Konditionierungsvariablen sind über alle Spezifikationen und Auswahl von Fällen hinweg hochgradig signifikant. Die weiter gehenden Befunde werden für die beiden abhängigen Variablen nacheinander diskutiert.

12 Andere Variablen, für die Joel Slemrod (2004) Niveaueffekte aufgrund theoretischer Überlegungen vermutet und empirisch bestätigt findet, müssen hier nicht weiter behandelt werden, da es sich um entwicklungspolitische Indikatoren handelt, die für die hier betrachteten OECD-Länder eine geringe Rolle spielen.

13 Dieser Indikator ist (für die hier betrachteten Länder) mit einem summarischen Indikator aller Arten von Kapitalverkehrsbeschränkungen hoch korreliert. Ich danke Christian W. Martin für die freundliche Überlassung dieser Daten; vgl. Martin (2004). 
Tabelle 2: $\quad$ Multivariate Regressionsanalysen zum Einfluss der Konditionierungsvariablen für das Jahr 2003

\begin{tabular}{|c|c|c|c|c|c|c|c|c|}
\hline \multirow{3}{*}{$\begin{array}{l}\text { Abhängige } \\
\text { Variable } \\
\text { Länder- } \\
\text { auswahl } \\
\text { Modell }\end{array}$} & \multicolumn{4}{|c|}{ Körperschaftsteuersatz (corprate) } & \multicolumn{4}{|c|}{ Spitzensteuersatz (topprate) } \\
\hline & \multicolumn{2}{|c|}{ OECD } & \multirow{2}{*}{\begin{tabular}{|c|}
$\begin{array}{c}\text { OECD/ } \\
\text { EU }\end{array}$ \\
$\mathbf{3}$ \\
\end{tabular}} & \multirow{2}{*}{$\begin{array}{c}\text { KPMG } \\
4\end{array}$} & \multicolumn{2}{|c|}{ OECD } & \multicolumn{2}{|c|}{ OECD/EU } \\
\hline & $\mathbf{1}$ & 2 & & & $\mathbf{1}$ & 2 & 3 & 4 \\
\hline $\begin{array}{l}\text { Unab- } \\
\text { hängige } \\
\text { Variablen }\end{array}$ & & & & & & & & \\
\hline $\begin{array}{l}\text { Konditio- } \\
\text { nierungs- } \\
\text { variable } \\
\text { (lnpop bzw. } \\
\text { totaltax) }\end{array}$ & $\begin{array}{c}3,71 * * * \\
(0,81)\end{array}$ & $\begin{array}{c}2,85 * * * \\
(0,59)\end{array}$ & $\begin{array}{c}2,54 * * * * \\
(0,85)\end{array}$ & $\begin{array}{c}2,89 * * * * \\
(0,51)\end{array}$ & $\begin{array}{c}0,60 * * * \\
(0,12)\end{array}$ & $\begin{array}{c}0,69 * * * \\
(0,13)\end{array}$ & $\begin{array}{c}0,57 * * * \\
(0,16)\end{array}$ & $\begin{array}{c}0,59 * * * \\
(0,14)\end{array}$ \\
\hline $\begin{array}{l}\text { Steuersatz } \\
\text { (topprate } \\
\text { bzw. } \\
\text { corprate) }\end{array}$ & $\begin{array}{l}0,34 * \\
(0,18)\end{array}$ & $\begin{array}{c}0,18 \\
(0,13)\end{array}$ & $\begin{array}{c}0,30 \\
(0,19)\end{array}$ & & $\begin{array}{c}0,14 \\
(0,13)\end{array}$ & $\begin{array}{c}0,04 \\
(0,15)\end{array}$ & $\begin{array}{c}0,28 * * \\
(0,12)\end{array}$ & $\begin{array}{c}0,22 * * \\
(0,10)\end{array}$ \\
\hline$g d p p c$ & $\begin{array}{l}-1,95 \\
(1,23)\end{array}$ & $\begin{array}{l}-1,40 \\
(0,86)\end{array}$ & $\begin{array}{c}1,09 \\
(1,08)\end{array}$ & $\begin{array}{l}1,30 * * \\
(0,54)\end{array}$ & $\begin{array}{l}2,62 * * \\
(0,92)\end{array}$ & $\begin{array}{c}1,75 \\
(1,07)\end{array}$ & $\begin{array}{l}1,96 * * \\
(0,77)\end{array}$ & $\begin{array}{c}3,09 * * * * \\
(0,72)\end{array}$ \\
\hline longcapl & & & & $\begin{array}{l}-2,17 \\
(1,39)\end{array}$ & & & & \\
\hline Konstante & $\begin{array}{c}-40,12 * * \\
(16,62)\end{array}$ & $\begin{array}{l}-18,62 \\
(12,47)\end{array}$ & $\begin{array}{c}-27,50 * * \\
(12,89)\end{array}$ & $\begin{array}{c}-18,66^{* *} \\
(8,30)\end{array}$ & $\begin{array}{l}12,24^{*} \\
(6,74)\end{array}$ & $\begin{array}{c}14,25^{* *} \\
(6,67)\end{array}$ & $\begin{array}{c}9,58 \\
(6,00)\end{array}$ & $\begin{array}{l}8,64 * \\
(5,02)\end{array}$ \\
\hline $\begin{array}{l}\text { Vernach- } \\
\text { lässigte } \\
\text { Ausreißer }\end{array}$ & - & Irland & - & - & - & Japan & - & $\begin{array}{c}\text { Luxem- } \\
\text { burg }\end{array}$ \\
\hline $\mathrm{N}$ & 21 & 20 & 32 & 63 & 21 & 20 & 32 & 31 \\
\hline $\begin{array}{l}\text { Bereinigtes } \\
\mathrm{R}^{2}\end{array}$ & 0,50 & 0,53 & 0,42 & 0,34 & 0,64 & 0,68 & 0,59 & 0,72 \\
\hline
\end{tabular}

Anmerkungen: Kleinste-Quadrate-Schätzung; zur Auswahl der Länder vgl. Text; Standardfehler in Klammern; zu den Definition der Variablen vgl. Text sowie Anhang; Signifikanzniveau: $* p<0,1, * * p<0,05$, *** $p<0,01$.

\section{Körperschaftsteuersatz}

Das Ergebnis in Modell 1 zeigt einen statistisch signifikanten Koeffizienten für den Spitzensteuersatz der persönlichen Einkommensteuer. Modell 2 zeigt jedoch, dass dieser »Effekt « weitgehend durch den irischen Fall erzeugt wird, der sich durch einen besonders niedrigen Körperschaftsteuersatz von 12,5\% auszeichnet. Nimmt man den irischen Fall heraus, halbiert sich der ohnehin kleine Koeffizient und ver- 
liert seine Signifikanz. Auch die Ergebnisse in Modell 3 - für »OECD/EU« - zeigen keinen signifikanten Effekt für den Spitzensteuersatz. Allerdings ist dabei zu beachten, dass Spitzensteuersatz und Wirtschaftskraft mit $r=0,60$ hoch korreliert sind. Vernachlässigt man jeweils eine der beiden Variablen - was hier nicht dargestellt wird -, so ist der Koeffizient der anderen Variablen größer und signifikant. ${ }^{14}$ Diese Ergebnisse reflektieren vor allem die Tatsache, dass sich die Beitrittsländer der EU sowohl durch eine geringere Wirtschaftskraft als auch durch niedrigere Steuersätze auszeichnen.

Dass Wirtschaftskraft eine Rolle spielt, wird von den Ergebnissen für »KPMG« gestützt, wobei allerdings Daten über die Spitzensteuersätze für diese Länderauswahl nicht vorhanden sind. ${ }^{15}$ Der Koeffizient für Wirtschaftskraft hat wiederum das richtige Vorzeichen (reiche Länder haben höhere Steuersätze), eine ähnliche Größenordnung und erreicht nun statistische Signifikanz. Interessanterweise ist der Koeffizient für Kapitalverkehrskontrollen nicht nur insignifikant, sondern hat auch das »falsche « Vorzeichen: Offenere Länder haben höhere Steuersätze. ${ }^{16}$

\section{Spitzensteuersatz}

Für »OECD« zeigt sich ein statistisch signifikanter Effekt für Wirtschaftskraft (Modell 1). Dieser Effekt wird etwas geringer und verliert seine Signifikanz, wenn man Japan als moderaten Ausreißer vernachlässigt (Modell 2). Die Regression für »OECD/EU « unterstützt jedoch die Schlussfolgerung, dass sich reichere Länder tendenziell höhere Spitzensteuersätze leisten (Modell 3). Auch wenn Wirtschaftskraft und Körperschaftsteuersatz gleichzeitig kontrolliert werden, ergeben sich für beide Variablen statistisch signifikante Koeffizienten. Der Effekt für den Körperschaftsteuersatz verkleinert sich, wenn man Luxemburg als multidimensionalen Ausreißer herauslässt, während sich gleichzeitig der Koeffizient für Wirtschaftskraft sowie die erklärte Varianz deutlich erhöhen (Modell 4). Trotzdem bleibt der Koeffizient des Körperschaftsteuersatzes signifikant. Modell 4 - das über $70 \%$ der Varianz in den Spitzensteuersätzen »erklärt« - impliziert, dass eine Senkung des Körperschaft-

14 Sieht man in der hohen Korrelation zwischen Wirtschaftskraft und Spitzensteuersätzen vor allem Multikollinearität, die letztlich durch zu wenige Fälle erzeugt wird, so liegt das Problem in der Unterschätzung der statistischen Signifikanz der Koeffizienten. Sieht man niedrigere Spitzensteuersätze dagegen als Konsequenz niedrigerer Wirtschaftskraft (Endogenität), so sind auch die Koeffizienten verzerrt eingeschätzt. M. E. handelt es sich letztendlich um eine Mischung aus beidem. Es ist plausibel, dass Wirtschaftskraft Körperschaftsteuersätze sowohl direkt als auch indirekt, also über die Spitzensteuersätze, beeinflusst, jedoch ist die Anzahl der Fälle zu gering, um die beiden Effekte klar zu trennen; vgl. auch Gary King (1991: 1050).

15 Die Daten für longcapl sind für einen Teil der von »KPMG« erfassten Länder (insbesondere osteuropäische Transformationsländer) nicht vorhanden, weshalb diese Länder in der entsprechenden Regression fehlen. Sie sind jedoch jedoch in der Regression für $» \mathrm{OECD} / \mathrm{EU}$ « erfasst.

16 Es ist zu beachten, dass die Zeitreihen für longcap1 nur bis zum Jahr 1998 reichen. Die Daten dieses Jahres werden in den Regressionen für das Jahr 2003 verwendet. 
steuersatzes um fünf Prozentpunkte in etwa mit einer Senkung des Spitzensteuersatzes um einen Prozentpunkt einhergeht. Es gibt also moderate Anzeichen dafür, dass niedrigere Körperschaftsteuersätze die Spitzensteuersätze der Einkommensteuer mit »hinunter ziehen«.

Insgesamt unterstützt die multivariate Analyse die uni- und bivariaten Analysen des vorangegangenen Abschnitts. Der Einfluss der beiden Konditionierungsvariablen - Ländergröße und Abgabenquote - ist robust, wenngleich in den größeren Ländergruppen auch andere Variablen Erklärungskraft besitzen. ${ }^{17}$ Ärmere Länder haben niedrigere Spitzensteuersätze als reichere, und es gibt Anzeichen dafür, dass der Wettbewerbsdruck bei der Körperschaftsteuer in die persönliche Einkommensteuer hinüberschwappt. Dieses »Überschwappen« ist jedoch bisher noch nicht besonders stark gewesen, und es zeigt sich vor allem bei Einbeziehung der EU-Beitrittsländer.

\section{Beta-Konvergenz und die Rolle von Vetopunkten}

In diesem letzten Teil der Konvergenzanalyse wird einerseits direkt getestet, ob absolute und/oder konditionale Beta-Konvergenz stattgefunden hat. Andererseits werden Vetopunkte und -spieler in die Analyse einbezogen (Immergut 1992; Huber et al. 1993; Lijphart 1999; Tsebelis 2002). Diese Einbeziehung ermöglicht einen weiteren, indirekten Test der Stärke struktureller Beschränkungen. Je stärker die Beschränkungen sind, desto eher kann man erwarten, dass sich policy-Präferenzen unterschiedlicher Vetospieler angleichen, weil die Kognitionen der Akteure einem starken Druck ausgesetzt sind (Ganghof 1999: 469, 2003: 9-13). Gleichen sich die policy-Präferenzen von Vetospielern an, so ist auch bei einer hohen Anzahl von Spielern policyWandel möglich (Tsebelis 2002: Kap. 2). ${ }^{18}$ Es ist deshalb zu erwarten, dass die Anzahl von Vetopunkten bei der Festlegung von Körperschaftsteuersätzen wesentlich weniger wichtig ist als bei der Festlegung der Spitzensteuersätze der persönlichen Einkommensteuer. Letztere sind keinem direkten Steuerwettbewerb ausgesetzt und die Regierungen können die relevante Konditionierungsvariable, die Abgabenquote, zumindest mittelfristig selbst beeinflussen. Der strukturelle Druck in Richtung Angleichung von policy-Präferenzen sollte somit geringer und die Rolle von Vetopunkten größer sein. Insbesondere ist folgende Annahme plausibel: Mit der Anzahl von Vetopunkten steigt die Wahrscheinlichkeit, dass Akteure, die gegen eine Senkung des Spitzensteuersatzes sind - typischerweise Mitte-Links-Parteien -, eine Vetoposition innehaben. Eine höhere Anzahl von Vetospielern sollte also ceteris paribus mit einer geringeren Senkung des Spitzensteuersatzes einhergehen.

17 Die gefundenen Muster stützen wiederum die Entscheidung, gesamtstaatliche Steuersätze zu betrachten. Bei der Betrachtung zentralstaatlicher Sätze finden sie sich nicht oder nur in geringerem Ausmaß.

18 Im Jargon der Vetospieler-Theorie kann also ein starker struktureller Druck auf die policy-Präferenzen der Akteure zu einer »Absorption« von Vetospielern führen; vgl. Tsebelis (2002: Kap. 2). 
Ich messe die Anzahl von Vetopunkten anhand von Arend Lijpharts (1999) Indikator für die Machtteilung in einem politischen System, d. h. seiner »FöderalismusUnitarismus«-Dimension (lijphart2) für den Zeitraum 1971-96. Diese Messung hat in erster Linie pragmatische Gründe: Lijpharts Index ist wohl der bekannteste einer Gruppe von - untereinander generell hoch korrelierten - Vetopunktindizes, wie sie etwa Uwe Wagschal (1999a, 1999b) in seinen Untersuchungen der Steuerpolitik benutzt hat.

Tabelle 3: Beta-Konvergenz und die konditionale Bedeutung von Vetopunkten

\begin{tabular}{|c|c|c|c|c|c|c|}
\hline \multirow{2}{*}{$\begin{array}{l}\text { Abhängige } \\
\text { Variable } \\
\text { Modell }\end{array}$} & \multicolumn{3}{|c|}{ corprate $_{2003}-$ corprate $_{1983}$} & \multicolumn{3}{|c|}{ topprate $_{2003}-$ topprate $_{1983}$} \\
\hline & 1 & 2 & 3 & 1 & 2 & 3 \\
\hline $\begin{array}{l}\text { Unabhängige } \\
\text { Variablen }\end{array}$ & & & & & & \\
\hline $\begin{array}{l}\text { Konditionie- } \\
\text { rungsvariable } \\
\text { (lnpop bzw. } \\
\text { totaltax) am } \\
\text { Periodenende }\end{array}$ & & $\begin{array}{c}3,44 * * * \\
(0,86)\end{array}$ & $\begin{array}{c}3,54 * * * \\
(1,02)\end{array}$ & & $\begin{array}{c}0,55 * * * \\
(0,14)\end{array}$ & $\begin{array}{c}0,65 * * * \\
(0,09)\end{array}$ \\
\hline $\begin{array}{l}\text { Abhängige } \\
\text { Variable am } \\
\text { Periodenanfang }\end{array}$ & $\begin{array}{c}-0,88 * * * \\
(0,18)\end{array}$ & $\begin{array}{c}-0,92 * * * \\
(0,14)\end{array}$ & $\begin{array}{c}-0,92 * * * \\
(0,14)\end{array}$ & $\begin{array}{c}-0,71 * * * \\
(0,11)\end{array}$ & $\begin{array}{c}-0,83 * * * \\
(0,09)\end{array}$ & $\begin{array}{c}-0,70 * * * \\
(0,06)\end{array}$ \\
\hline $\begin{array}{l}\text { Vetopunkte } \\
\text { (lijphart2) }\end{array}$ & & & $\begin{array}{l}-0,21 \\
(1,06)\end{array}$ & & & $\begin{array}{c}2,85 * * * \\
(0,60)\end{array}$ \\
\hline Konstante & $\begin{array}{c}-26,54 * * * \\
(9,24)\end{array}$ & $\begin{array}{l}-29,13 * \\
(15,49)\end{array}$ & $\begin{array}{l}-30,69 \\
(17,80)\end{array}$ & $\begin{array}{c}28,08 * * * \\
(7,25)\end{array}$ & $\begin{array}{c}15,28 * * \\
(6,24)\end{array}$ & $\begin{array}{c}1,98 \\
(5,05)\end{array}$ \\
\hline $\mathrm{N}$ & 21 & 21 & 21 & 21 & 21 & 21 \\
\hline Bereinigtes $\mathrm{R}^{2}$ & 0,52 & 0,73 & 0,72 & 0,68 & 0,82 & 0,92 \\
\hline
\end{tabular}

Anmerkungen: Kleinste-Quadrate-Schätzung; zur Auswahl der Länder vgl. Text; Standardfehler in Klammern; zur Definition der Variablen vgl. Text sowie Anhang; Signifikanzniveau: * $p$ $<0,1, * * p<0,05$, *** $p<0,01$.

Eine Alternative wäre eine Dummy-Variable, die den Unterschied zwischen Einparteienregierungen und anderen einfängt (Hallerberg/Basinger 1998; Tsebelis 2002: 203). Ich vernachlässige diese Alternative hier auch aus zwei theoretischen Gründen: Erstens halte ich die generelle Gleichsetzung von Einparteienregierungen und Ein-Vetospieler-Systemen für unplausibel; Einparteien-Minderheitsregierungen müssen im Parlament den Kompromiss mit mindestens einer weiteren Partei oder unabhängigen Abgeordneten suchen (vgl. Ganghof/Bräuninger 2005). Zweitens ist es für die verfolgte Fragestellung von Vorteil, dass Lijphart föderalstaatliche Strukturen berücksichtigt. Zum einen betrachten wir gesamtstaatliche Steuersätze, sodass 
die eventuell vorhandene Vetomacht subnationaler Ebenen einbezogen werden sollte (vgl. Ganghof 1999: 466). Zum anderen fängt Lijpharts Index indirekt die Macht von gut organisierten innerparteilichen Gruppierungen ein, die gerade bei Einparteienregierungen eine wichtige Rolle spielen und auch nach dem Ansatz von George Tsebelis (2002) berücksichtigt werden sollten. Entscheidend ist, dass die Macht dieser Gruppierungen ihr Fundament häufig auf gliedstaatlicher Ebene hat. Ein Beispiel dafür ist Australien, wo etwa die factions der Australian Labor Party ihre Macht aus den gliedstaatlichen Parteiorganisationen ableiten (Nagel 1998: 265; vgl. auch Mulé 2001).

Tabelle 3 stellt die Ergebnisse von Regressionen dar, welche die Veränderung der Steuersätze zwischen 1983 und 2003 (corprate 2003 - corprate $_{1983}$ bzw. topprate 2003 - topprate 1983 ) mithilfe von drei Variablen erklären:

- der jeweiligen Konditionierungsvariable (lnpop bzw. taxtotal) zum Periodenende (2003);

- der Konvergenzvariable, also der abhängigen Variable zum Periodenanfang (1983);

- der Vetopunkte-Variable (lijphart2). ${ }^{19}$

Bei der Interpretation der Ergebnisse ist zu beachten, dass sich eine Steuersatzsenkung in negativen Werten der abhängigen Variablen ausdrückt. Wir erwarten deshalb für die Konvergenzvariable einen negativen und für die anderen beiden Variablen positive Koeffizienten.

Die Ergebnisse bestätigen unsere Hypothesen. Betrachten wir zunächst die Veränderung der Körperschaftsteuersätze. ${ }^{20}$ Drei Resultate sind hier wichtig:

(1) Es hat absolute Beta-Konvergenz stattgefunden (Modell 1). In der bivariaten Regression ist der Beta-Koeffizient des Ausgangssteuersatzes negativ, relativ groß und hochgradig signifikant. Jeder Prozentpunkt, den der Steuersatz im Jahr 1983 höher lag, führte zu einer um etwa 0,9 Prozentpunkte höheren Steuersatzsenkung. Wir wissen jedoch aus der obigen Analyse, dass diese starke Beta-Konvergenz nur zu einer schwachen Sigma-Konvergenz geführt hat. Der Beta-Koeffizient für den Ausgangssteuersatz spiegelt also auch Positionswechsel einzelner Länder innerhalb der Steuersatzverteilung wider. ${ }^{21}$

(2) Dieser Positionswechsel einzelner Länder ist zum Teil auch das Ergebnis davon, dass sich die Steuersätze zunehmend an der Ländergröße ausgerichtet haben (Modell 2). Berücksichtigt man diese Variable im Regressionsmodell, steigert sich die erklärte Varianz deutlich, und der Beta-Koeffizient des Ausgangssteuersatzes erhöht sich ebenfalls geringfügig. Es hat also konditionale Konvergenz stattgefunden.

19 Regressionen für kürzere Zeiträume und andere Vetopunktindizes erbringen qualitativ identische Ergebnisse.

20 Irland ist in diesen Regressionen aufgrund des seit 2003 niedrigen Körperschaftsteuersatzes von 12,5\% ein moderater Ausreißer. Nimmt man Irland aus der Fallauswahl heraus, werden die hier präsentierten Ergebnisse gestärkt, und die erklärte Varianz steigt zum Teil auf ca. $85 \%$.

21 Vgl. dazu auch die Kritik am Konzept der Beta-Konvergenz von Milton Friedman (1992) und Dennis Quah (1993). 
(3) Die Anzahl der Vetopunkte hat im Konvergenzprozess keine Rolle gespielt. Der Koeffizient für die Vetopunkt-Variable ist klein, hat das falsche Vorzeichen, ist insignifikant und verschlechtert die »Erklärungsleistung « des Regressionsmodells (Modell 3).

In Bezug auf die Spitzensteuersätze sind ebenfalls drei Resultate beachtenswert:

(1) Es hat absolute Beta-Konvergenz stattgefunden (Modell 1). Im bivariaten Modell ist der Beta-Koeffizient des Ausgangssteuersatzes negativ, relativ groß und hochgradig signifikant. Dies kann auch gar nicht anders sein, weil Beta-Konvergenz eine notwendige Bedingung für die oben diagnostizierte Sigma-Konvergenz ist.

(2) Wiederum hat auch konditionale Konvergenz stattgefunden (Modell 2). Bezieht man die Abgabenquote als unabhängige Variable ein, erhöhen sich sowohl die erklärte Varianz als auch der Regressionskoeffizient des Ausgangssteuersatzes deutlich. Niedrigsteuerländer haben also ceteris paribus stärkere Steuersenkungen beschlossen als Hochsteuerländer.

(3) Die Anzahl der Vetopunkte hat im Konvergenzprozess eine wichtige Rolle gespielt (Modell 3). Die Integration der Vetopunkt-Variable erhöht die erklärte Varianz erheblich und der Beta-Koeffizient der Variablen ist hochgradig signifikant. Seine Größe impliziert, dass ein Land mit vielen Vetopunkten wie Deutschland eine um etwa zehn Prozentpunkte niedrigere Steuersatzsenkung beschlossen hat als ein Land mit wenigen Vetopunkten wie Großbritannien. ${ }^{22}$

Auch diese Ergebnisse stehen im Einklang mit den Befunden vergleichender Fallstudien. Die policy-Präferenzen der Vetospieler haben sich in Bezug auf die Körperschaftsteuer nicht nur zwischen den Ländern, sondern auch innerhalb der Länder angeglichen, sodass eine Einigung auf Steuersatzsenkungen in der Regel auch vielen Vetospielern nicht schwer gefallen ist. Anders bei der persönlichen Einkommensteuer: Hier gab es klare Rechts-Links-Gegensätze, sodass linke Parteien mit Vetomacht in der Lage waren, Steuersatzsenkungen zu verhindern oder zu verringern. Die besten Beispiele sind Australien und Deutschland, wo Parteien links von Mitte-Rechts-Regierungen Senkungen des Spitzensteuersatzes verhindert haben (Ganghof 2004; Ganghof/Eccleston 2004).

Natürlich werden die Effekte von Vetoinstitutionen in der Regressionsanalyse nur grob eingefangen. Die komplexe Interaktion zwischen Status quo, der Anzahl von Vetospielern und ihren Präferenzen ist theoretisch leichter zu modellieren als empirisch zu messen (Tsebelis 2002). Fallstudien liefern hier wichtige Einsichten. Sie zeigen vor allem, dass eine höhere Anzahl von Vetospielern unter Umständen auch zu einer stärkeren Senkung des Steuersatzes führen kann. Dies gilt insbesondere dann, wenn die Regierung den Status quo beim Körperschaftsteuersatz dringend verändern will und dazu die Zustimmung eines rechts von ihr platzierten Vetospielers benötigt. So haben etwa die schwedischen Liberalen der sozialdemokratischen Minderheitsregierung Anfang der neunziger Jahre weit reichende Satzsenkungen bei der persönlichen Einkommensteuer abgerungen; genauso hat sich die bürgerliche

22 Die jeweiligen Werte auf Lijpharts Skala sind 2,53 für Deutschland und -1,19 für Großbritannien (Lijphart 1999: 312). 
Opposition im deutschen Bundesrat gegenüber der rot-grünen Regierung Ende der neunziger Jahre verhalten (Salsbäck 1993; Ganghof 2004). Diese Befunde ergänzen die quantitative Analyse und bestätigen das Ergebnis, dass Vetospieler in der persönlichen Einkommensteuer weiterhin eine wichtige Rolle spielen.

Zusammenfassend kann man in doppelter Weise von konditionaler Konvergenz sprechen: Die Konvergenz der Steuersätze wird von strukturellen Konditionierungsvariablen bedingt und die Konvergenz der Vetospieler-Präferenzen hängt systematisch von der Stärke der strukturellen Beschränkungen ab. Je schwächer und diffuser diese Beschränkungen sind, desto geringer ist die Konvergenz der Präferenzen und desto wichtiger die Anzahl der Vetopunkte bzw. -spieler.

\section{Schlussfolgerungen}

Das Ziel dieses Beitrags war es, zu einem besseren Verständnis der strukturellen Beschränkungen von Einkommensteuerreformen in OECD-Staaten beizutragen. Dieses Verständnis ist nicht nur für aktuelle Reformdiskussionen relevant (vgl. Ganghof 2004: Kap. 5), sondern auch notwendig, um unterschiedliche Erklärungen weltweiter Reformtrends angemessen einzuschätzen. Ich werde zunächst die zentralen Ergebnisse zusammenfassen und ihre generelle Relevanz für ideenorientierte Erklärungen diskutieren. Anschließend gehe ich kurz auf die Frage ein, welche Auswirkungen die Abwärtstrends bei den Steuersätzen auf die staatliche Aufgabenerfüllung haben können.

\subsection{Ursachen der Abwärtstrends}

Die Abwärtsspirale bei den Körperschaftsteuersätzen wurde in großem Maße vom Steuerwettbewerb angetrieben. Hohe tarifliche Steuersätze sind ein erheblicher Nachteil im internationalen Standortwettbewerb, da sie profitable Investitionen besonders stark treffen und multinationale Unternehmen dazu veranlassen, Gewinne in Niedrigsteuerländer zu verlagern. Wie wichtig der Steuerwettbewerb war, zeigt sich nicht nur in Fallstudien von Steuerreformprozessen, sondern auch auf aggregierter Ebene. Die Steuersätze haben sich zunehmend an der Ländergröße ausgerichtet, wie dies die Theorie des asymmetrischen Steuerwettbewerbs vorhergesagt hat. Ideenorientierte Erklärungen des Abwärtstrends bei den Körperschaftsteuersätzen müssen deshalb qualifiziert werden. Die innerstaatlichen »Lernanreize« deuteten nicht eindeutig in Richtung einer drastischen Senkung der Körperschaftsteuersätze. Insbesondere linke Parteien hatten einen Anreiz, hohe tarifliche Steuersätze beizubehalten, um so besonders profitable Unternehmen weiterhin hoch zu besteuern und die Funktion der Körperschaftsteuer als »Auffangbecken « der persönlichen Einkommensteuer zu stärken. Der intensive Steuerwettbewerb erklärt, warum sich die Steuersatz-Präferenzen linker Parteien trotzdem an die rechter Parteien angeglichen haben. Weil diese Konvergenz von Präferenzen stattgefunden hat, spielt die Anzahl 
von Vetospielern und -punkten für die Festlegung von Körperschaftsteuersätzen praktisch keine Rolle.

Der Abwärtstrend bei den Spitzensteuersätzen der persönlichen Einkommensteuer kann nicht mit einem direkten Wettbewerbsdruck erklärt werden. Insofern ist die Skepsis von Verfechtern ideenorientierter Sichtweisen gegenüber dem Buhmann (bogey-man) »Globalisierung « berechtigt. Die alternative Erklärung dieser Autoren bleibt jedoch vage, weil sie die strukturellen Beschränkungen auf innerstaatlicher Ebene ebenfalls nicht systematisch berücksichtigt. Die zentrale Beschränkung für die Absenkung der Steuersätze auf hohe (Arbeits-)Einkommen liegt in der Gesamtsteuerlast des Faktors Arbeit. Ist diese hoch, so führt eine starke Absenkung der Steuersätze entweder zu größeren Einnahmeausfällen oder zu einer erheblich verringerten Progression in der Lohnbesteuerung insgesamt. Entsprechend dieser Logik zeigt sich ein statistischer Zusammenhang zwischen Gesamtabgabenquote und Spitzensteuersatz, der trotz des Abwärtstrends bei den Spitzensteuersätzen erhalten geblieben ist. Anders als bei der Körperschaftsteuer haben also Hochsteuerländer bei der Einkommensteuer systematisch höhere Spitzensteuersätze beibehalten.

\section{Abbildung 1: Gesamtsteueraufkommen und Einkommensteuersätze}

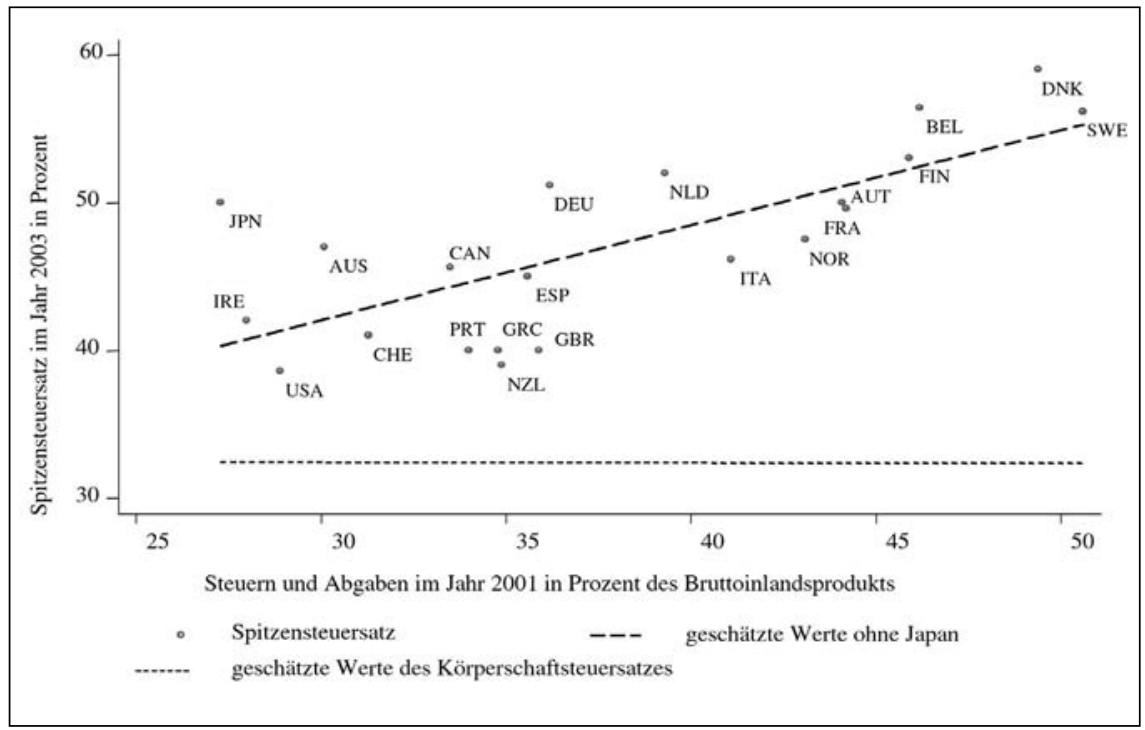

Quellen: Siehe Anhang.

Abbildung 1 veranschaulicht diesen Zusammenhang für das Jahr 2003, also das Ende des hier betrachteten Zeitraums. Das Streudiagramm setzt die Abgabenquoten der OECD-Länder zu ihren Spitzensteuersätzen in Beziehung. Neben der dazugehörigen Regressionsgerade ist auch die entsprechende Gerade für die Körperschaft- 
steuersätze eingezeichnet. ${ }^{23}$ Man sieht, dass Hochsteuerstaaten keine höheren Körperschaftsteuersätze haben als Niedrigsteuerstaaten, sehr wohl aber höhere Spitzensteuersätze. Der Preis, den sie dafür zahlen müssen, ist, dass die Spitzensteuersätze ausschließlich oder überwiegend für Arbeitseinkommen gelten.

Anhand der Abbildung lässt sich der Zusammenhang zwischen Reformideen und strukturellen Beschränkungen verdeutlichen. Wenn der dänische Spitzensteuersatz von 59\% heute 20 Prozentpunkte über dem neuseeländischen angesiedelt ist, so liegt dies zweifellos daran, dass sich in Neuseeland radikalere Steuerreformideen durchgesetzt haben (Ganghof 2004: 132-36). Die neuseeländischen Reformer haben sich am Ideal einer umfassenden Einkommensteuer orientiert, die alle Arten von Einkommen gleich behandelt. Der Spitzensteuersatz der persönlichen Einkommensteuer wurde möglichst stark abgesenkt, damit er auch auf sensible Arten von Kapitaleinkommen angewendet werden kann. Die dänischen Reformer haben dagegen die Steuerlast auf Kapital und Arbeit stark differenziert und hohe Steuersätze auf persönliche Einkommen, vor allem auf Arbeitseinkommen, beibehalten. Dieser Unterschied in den umgesetzten Reformideen basiert jedoch in großem Maße auf dem Unterschied in der Gesamtsteuerlast. In Neuseeland konnten sich radikalere Ideen über die Struktur der Einkommensteuer auch deshalb durchsetzen, weil dort das Niveau der gesamten Steuer- und Abgabenlast geringer war als in Dänemark.

Allerdings sind die strukturellen Beschränkungen einer Reform der persönlichen Einkommensteuer durch die Abgabenbelastung weniger eindeutig und rigide als die zuvor konstatierten Beschränkungen des internationalen Steuerwettbewerbs für die Körperschaftsteuer. Die Regierungen haben die Steuerlast auf den Faktor Arbeit zumindest mittel- und langfristig selbst unter Kontrolle - was für Steuerwettbewerb und Ländergröße nicht gilt. Sie können sich also dafür entscheiden, die Steuerlast mittelfristig stark abzusenken oder die Progression der Einkommensbesteuerung von Löhnen zu reduzieren. Aus diesem Grund bleiben parteipolitische Unterschiede bestehen und sind auch Vetopunkte oder -spieler für die Festlegung von Einkommensteuersätzen von Bedeutung. Dies hat sich in den Regressionsanalysen gezeigt und kann anhand einzelner Fallstudien nachvollzogen werden. Wenn etwa Neuseeland in Abbildung 1 deutlich unterhalb der Regressionsgeraden liegt und Australien deutlich darüber, so liegt dies nicht zuletzt daran, dass nur in Australien linksgerichtete Vetospieler vorhanden waren, die weiter gehende Senkungen des Spitzensteuersatzes verhinderten (Ganghof/Eccleston 2004; Ganghof 2005).

Die vorgelegte Analyse modifiziert somit prominente ideenorientierte Erklärungen von Einkommensteuerreformen. Letztere bleiben meines Erachtens aus zwei zentralen Gründen unbefriedigend: Erstens versäumen sie es, systematisch zu bestimmen, welche Ideen in nationalen Reformdiskussionen potenziell hätten Bedeutung erlangen können. Da sie sich nur auf diejenigen konkreten Reformmodelle konzentrieren, die tatsächlich relevant geworden sind, können diese Ansätze zwar erklären,

23 Der Übersichtlichkeit halber sind die tatsächlichen Körperschaftsteuersätze nicht dargestellt. $\mathrm{Zu}$ beachten ist auch, dass der japanische »Ausreißer« bei der Berechnung der Regressionsgeraden für die Spitzensteuersätze unberücksichtigt bleibt, um den Zusammenhang für die restlichen Länder klarer zu machen. 
warum es überhaupt zu policy-Wandel kam, aber nicht, in welche Richtung dieser Wandel fortschritt oder welches Ausmaß er hatte. Autoren wie Steinmo, Swank oder Garrett argumentieren, dass die Einkommensbesteuerung von der allgemeinen Idee »marktkonformer « Besteuerung beeinflusst wurde, aber sie analysieren nicht systematisch die verschiedenen Optionen marktkonformer Einkommensbesteuerung. ${ }^{24}$ Die Frage, warum bestimmte Reformoptionen nicht verfolgt wurden, gerät deshalb gar nicht erst in ihr Blickfeld.

Um die Frage zu beantworten, müssen zweitens die materiellen Beschränkungen, denen sich die Entscheidungsträger gegenüber sahen, systematisch berücksichtigt werden. Der Grund liegt letztlich darin, dass Kognitionen (beliefs) nicht beobachtbar sind. Sozialwissenschaftler müssen von schriftlichen oder mündlichen Äußerungen der Akteure auf deren Kognitionen schließen. Solche Schlüsse sind aber angesichts der vielfältigen Interpretationsmöglichkeiten überhaupt nur dann möglich, wenn der Interpretationsspielraum »extern« begrenzt wird - wenn man also die Äußerungen der Akteure zu den Beschränkungen in Beziehung setzt, denen sie sich gegenübersehen (vgl. auch Bartelborth 1999; Bartelborth/Scholz 2002), und man so ihren »Platz in der Welt« ermittelt (Dennett 1987: 17). Auch diese systematische Analyse materieller Möglichkeiten und Beschränkungen ist bei den Verfechtern ideenorientierter Erklärungen von Steuerreformen zu kurz gekommen. Steinmos These etwa, wonach ökonomische »Globalisierung « Steuersatzsenkungen nur gerechtfertigt, aber nicht verursacht hat, muss angesichts der hier vorgelegten Analyse zurïckgewiesen werden. Steuerwettbewerb bei der Körperschaftsteuer ist für die nationalen politischen Entscheidungsträger Realität.

\subsection{Die Auswirkungen des Steuerwettbewerbs}

Welche Auswirkungen haben die Befunde für die Entscheidungsfreiheiten nationalstaatlicher Politik bei der Einkommensbesteuerung? Drei Implikationen sollen hier diskutiert werden:

(1) Die erste betrifft die Besteuerung von Kapitalgesellschaften. Ein Grund dafür, dass sich die policy-Präferenzen der Entscheidungsträger so schnell einem System mit niedrigen Steuersätzen und breiter Bemessungsgrundlage annäherten, war, dass Senkungen der Körperschaftsteuersätze nicht unbedingt zu niedrigen Steuereinnahmen führen müssen. Fast alle Regierungen haben steuerliche Investitionsanreize, z. B. die »beschleunigte Abschreibung « von Investitionsgütern, abgebaut, um die Steuersatzsenkungen zu finanzieren. Deshalb ist der Steuerwettbewerb aber nicht folgenlos. Ohne Wettbewerbsdruck hätten viele - wenngleich sicher nicht alle - Regierungen diese Investitionsanreize wahrscheinlich beibehalten, oder sie hätten sich stärker und systematischer in Richtung einer direkten Ausgabensteuer bewegt. Anders formuliert: Sie hätten

24 Die allgemeine Idee »marktkonformer« Einkommensbesteuerung wird somit nicht hinreichend genau bestimmt, obwohl diese Bestimmung der Ausgangspunkt jeder derartigen Untersuchung sein sollte (Jachtenfuchs 1995: 431). 
eine Form der Körperschaftsbesteuerung gewählt, die Reingewinne höher besteuert als normale Kapitaleinkommen (vgl. auch Det Økonomiske Råd 2001: Kap. 1).

Angesichts des Steuerwettbewerbs war dies jedoch nicht mehr möglich. Wenn also stimmt, was die vergleichende politische Ökonomie lange postuliert hat, nämlich dass ein System mit höheren Steuersätzen und einer investitionsfreundlichen Bemessungsgrundlage effizienter ist als ein System mit niedrigen Steuersätzen (Wallerstein/Przeworki 1995), dann hat der Steuerwettbewerb die Besteuerung der Kapitalgesellschaften ineffizienter gemacht, zumindest in Bezug auf die bereits im Land ansässigen Unternehmen (vgl. Bond 2000; Haufler/Schjelderup 2000). Um diese verringerte Besteuerungseffizienz auszugleichen, müsste die Durchschnittsbelastung der Kapitalgesellschaften mittel- und langfristig sinken. Ob es dazu kommt und wie stark dieser Effekt sein wird, bleibt abzuwarten. Klar ist jedoch, dass sich das Muster aufkommensneutraler Steuersatzsenkungen in Zukunft nicht ohne weiteres fortsetzen lässt.

(2) Die zweite Auswirkung betrifft den Zusammenhang zwischen Körperschaftsteuer und persönlicher Einkommensteuer. Aus meinen Ergebnissen folgt, dass die Nationalstaaten bisher trotz Steuerwettbewerb in der Lage waren, ihre eigentlichen steuerpolitischen Ziele weiterhin zu erreichen. Dazu konnten sie jedoch nicht einfach ihre bisherige Politik beibehalten. Stabilität in der Zielerreichung setzte vielmehr policy-Wandel, also die Veränderung des legislativen Status quo, voraus. Regierungen mussten ihre Einkommensteuersysteme umfassend umstrukturieren, um die angestrebten materiellen Ergebnisse, also etwa Einnahmen oder Progressivität, weiterhin zu erreichen. Dies ist analytisch von großer Bedeutung, weil sich dadurch für die nationalen Vetoakteure die Rückfall-Option (default condition) also das Ergebnis, das eintritt, wenn sich die Akteure nicht auf eine Änderung des Status quo einigen können - änderte.

Auf welche Weise sich damit die innerstaatliche Machtbalance ändern kann, zeigt insbesondere der deutsche Fall (vgl. ausführlich: Ganghof 2004). Ähnlich wie andere sozialdemokratische Parteien auch hatte die SPD kein Interesse daran, die Einnahmen oder die Progressivität der Einkommensteuer zu verringern. Ohne Wettbewerbsdruck hätte sie einfach auf dem Status quo beharren können - so wie sie dies 1997/98 gegenüber der Regierung Kohl getan hat. Angesichts der dringenden Notwendigkeit, den rechtlichen Status quo zu verändern - insbesondere durch eine drastische Senkung des Körperschaftsteuersatzes -, war dies jedoch nach der Regierungsübernahme im Jahr 1998 keine Option mehr. Die Wettbewerbsdimension wurde im steuerpolitischen policy-Raum so bedeutend, dass die rot-grüne Regierung eine Steuerreform dringend brauchte. Dies brachte die anderen Vetoakteure, also das Verfassungsgericht und den Bundesrat, in die Position, der Regierung weit reichende Konzessionen abverlangen zu können. Letzteres wiederum hat erheblich dazu beigetragen, dass in Deutschland - anders als in vergleichbaren Ländern unter einer Mitte-Links-Regierung eine weitgehende Senkung des Spitzensteuersatzes und eine deutliche Nettoentlastungen bei der Einkommensteuer umgesetzt wurde. Im Zusammenspiel mit der innerstaatlichen institutionellen Machtverteilung kann der Steuerwettbewerb bei den Kapitalgesellschaften also durchaus erhebliche 
Auswirkungen haben - auch und gerade für das Niveau und die Progressivität der persönlichen Einkommensbesteuerung.

(3) Die dritte Implikation knüpft an das deutsche Beispiel an. Einerseits ist der deutsche Fall im internationalen Vergleich untypisch. Die meisten Länder haben sich grundsätzlich von der Idee verabschiedet, den Spitzensteuersatz der persönlichen Einkommensteuer am Körperschaftsteuersatz auszurichten. Deshalb hatte der Körperschaftsteuerwettbewerb für die persönliche Einkommensteuer geringere Auswirkungen als hierzulande. Andererseits ist eine gewisse »Sogwirkung « niedriger Körperschaftsteuersätze auf die Spitzensteuersätze der Einkommensteuer generell erkennbar. Anzeichen dafür wurden in der quantitativen Analyse gefunden, und das Bild in Fallstudien ist noch deutlicher (Ganghof/Eccelston 2004; Ganghof 2005; Hobson 2003). In Ländern, in denen eine große Lücke zwischen den beiden Steuersätzen klafft, spielt diese im policy-Diskurs eine wichtige Rolle. Da die Lücke eine offensichtliche Diskriminierung von Arbeitseinkünften und ein Hemmnis für durchgreifende Steuervereinfachung darstellt, kann sie von Befürwortern niedriger und stark abgeflachter Einkommensteuern mit Erfolg angeprangert werden.

Niedrige Körperschaftsteuersätze stärken letztlich die Befürworter einer »Flat Tax «, wie sie etwa die Slowakei vor kurzem eingeführt hat. Solch eine Einkommensteuer zeichnet sich vor allem dadurch aus, dass es nur einen einheitlichen und moderaten Steuersatz - in der Slowakei 19\% - gibt, der dem Körperschaftsteuersatz entspricht. So unwahrscheinlich es ist, dass die Slowakei kurzfristig viele Nachahmer innerhalb der OECD findet, so wahrscheinlich ist es, dass der Trend zu flacheren Einkommensteuertarifen weitergeht. Denn nur so können Regierungen die Lücke zwischen dem Spitzensteuersatz der Einkommensteuer und dem Körperschaftsteuersatz möglichst gering halten. Ich kann mich somit Musgraves eingangs zitierter Diagnose und Vorhersage zumindest teilweise anschließen: Der Steuerwettbewerb trägt zur Abflachung von Einkommensteuern bei. Der Effekt ist zwar indirekt, aber unbestreitbar. 


\section{Anhang: Definition und Quellen der Variablen}

\subsection{Abhängige Variablen}

corprate: Nominaler Körperschaftsteuersatz auf einbehaltene Gewinne in Prozent. Bei progressiven Steuertarifen wird der höchste Steuersatz erfasst. Lokale Steuern (auch Gewerbesteuern) und Zuschläge werden berücksichtigt, ebenso die Abzugsfähigkeit der Steuern von ihrer eigenen Bemessungsgrundlage oder der anderer Steuern. Die Steuersätze der OECD-Länder stammen aus folgenden Quellen: Coopers\&Lybrand International Tax Summaries, ${ }^{25}$ KPMG Corporate Tax Survey, ${ }^{26}$ $»$ Die wichtigsten Steuern im internationalen Vergleich« (erstellt vom Bundesfinanzministerium), ${ }^{27}$ Devereux et al. (2002), Office of Tax Policy Research (2004) sowie in Einzelfällen nationalen Quellen. Die Steuersätze für die EU-Beitrittsländer stammen ebenfalls vom Bundesfinanzministerium (»Die wichtigsten Steuern im internationalen Vergleich«). Die Steuersätze für alle anderen Länder entsprechen denen des KPMG Corporate Tax Survey und sind beim Autor einzusehen.

topprate: Nominaler Spitzensteuersatz in Prozent. Lokale Steuern und Zuschläge werden berücksichtigt, ebenso die Abzugsfähigkeit der Steuern von ihrer eigenen Bemessungsgrundlage oder der anderer Steuern. Bei getrennten Steuertarifen für Arbeits- und Kapitaleinkommen wird der Steuersatz für Arbeitseinkommen betrachtet. Die Steuersätze entstammen den oben angegebenen Quellen sowie Personal Income Tax Systems under Changing Economic Conditions ${ }^{28}$ der OECD aus dem Jahr 1986. Fehlende Werte für die Schweiz und Kanada sind interpoliert.

\subsection{Unabhängige Variablen}

gdppc: Bruttoinlandsprodukt pro Kopf in 10.000 US-Dollar. Die Daten sind der World Development Indicators CD-ROM 2003 der Weltbank entnommen und über den Autor zugänglich. Für die Jahre 2002 und 2003 wird der Wert des Jahres 2001 fortgeschrieben.

lijphart2: Anzahl von Vetopunkten, gemessen durch Lijpharts (1999) Index der Machtteilung in politischen Systemen (»Föderalismus-Unitarismus-Dimension«) für den Zeitraum 1971-1996. Hohe Werte zeigen eine hohe Anzahl von Vetopunkten an.

lnpop: Natürlicher Logarithmus der Bevölkerungszahl (in Millionen). Die Daten stammen aus der World Development Indicators CD-ROM 2003 der Weltbank und sind über den Autor zugänglich. Für die Jahre 2002 und 2003 wird der Wert des Jahres 2001 fortgeschrieben.

25 Aus verschiedenen Jahren; zugänglich über den Autor.

26 Aus verschiedenen Jahren; zugänglich in: http://www.us.kpmg.com/microsite/global_ tax/ctr_survey/; 11.1.2004.

27 Aus verschiedenen Jahren; zugänglich über den Autor.

28 Zugänglich über den Autor. 
loncap1: Indikator der Beschränkung ausländischer Direktinvestitionen. Er kann die Werte 0, 1 und 2 annehmen, wobei höhere Werte stärkere Beschränkungen abbilden. Die Daten stammen aus den Reports on Exchange Arrangements and Exchange Restrictions ${ }^{29}$ des Internationalen Währungsfonds und wurden mir freundlicherweise von Christian W. Martin überlassen (vgl. Martin 2004). Das letzte verfügbare Jahr ist 1998, sodass die entsprechenden Werte in der Regressionsanalyse bis 2003 fortgeschrieben werden.

taxtotal: Steuern und Abgaben in Prozent des Bruttoinlandsprodukts. Es wird jeweils der Wert für das letzte verfügbare Jahr fortgeschrieben. Für die Mitgliedsstaaten der OECD stammen die Daten aus dem OECD Statistical Compendium. ${ }^{30}$ Für andere Länder basieren die Daten auf Länderstudien des Internationalen Währungsfonds sowie nationalen Quellen. Bei fehlenden Werten wird jeweils das letzte verfügbare Jahr berücksichtigt, in der Regel das Jahr 2001.

29 Zugänglich über den Autor.
30 Zugänglich über den Autor. 


\section{Literatur}

Bartelborth, Thomas 1999: Verstehen und Kohärenz. Ein Beitrag zur Methodologie der Sozialwissenschaft, in: Analyse \& Kritik 21: 1, 97-116.

Bartelborth, Thomas/Scholz, Oliver Robert 2002: Understanding Utterances and Other Actions, in: Grewendorf, Günther/Meggle, Georg (Hrsg.): Speech Acts, Mind, and Social Reality: Discussions with John Searle, London, 165-186.

Boadway, Robin/Bruce, Neil 1984: A General Proposition on the Design of a Neutral Business Tax, in: Journal of Public Economics 24: 1, 231-239.

Bond, Stephen R. 2000: Levelling Up or Levelling Down? Some Reflections on the ACE and CBIT Proposals, and the Future of the Corporate Tax Base, in: Cnossen, Sijbren (Hrsg.): Taxing Capital Income in the European Union, Oxford, 161-179.

Bordignon, Massimo/Giannini, Silvia/Panteghini, Paolo 2001: Reforming Business Taxation: Lessons from Italy?, in: International Tax and Public Finance 8: 2, 191-210.

Brooks, Stephen G./Wohlforth, William C. 2000: Power, Globalization and the End of the Cold War: Reevaluation a Landmark Case for Ideas, in: International Security 25: 3, 5-53.

Brooks, Stephen G./Wohlforth, William C. 2002: From Old Thinking to New Thinking in Qualitative Research, in: International Security 26: 4, 93-111.

Bucovetsky, Sam 1991: Asymmetric Tax Competition, in: Journal of Urban Economics 30: 2, 167-181.

Dehejia, Vivek H./Genschel, Philipp 1999: Tax Competition in the European Union, in: Politics and Society 27: 3, 304-430.

Dennett, Daniel C. 1987: The Intentional Stance, Cambridge, MA.

Det Økonomiske Råd 2001: Danish Economy: Spring 2001 (English Summary), in: http:// www.dors.dk/rapp/dors086.htm; 12.10.2002.

Devereux, Michael P./Griffith, Rachel 1998: Taxes and the Location of Production: Evidence from a Panel of US Multinationals, in: Journal of Public Economics 68: 3, 335-367.

Devereux, Michael P./Griffith, Rachel 2003: Evaluating Tax Policy for Location Decisions, in: International Tax and Public Finance 10: 2, 107-126.

Devereux, Michael P./Griffith, Rachel/Klemm, Alexander 2002: Corporate Income Tax Reforms and International Tax Competition, in: Economic Policy 17: 35, 451-495.

Fearon, James/Wendt, Alexander 2002: Rationalism v. Constructivism: A Skeptical View, in: Carlsnaes, Walter/Risse, Thomas/Simmons, Beth A. (Hrsg.): Handbook of International Relations, London, 52-72.

Friedman, Milton 1992: Do Old Fallacies Never Die?, in: Journal of Economic Literature 30: 4, 2129-2132.

Fuest, Clemens/Weichenrieder, Alfons 2002: Tax Competition and Profit Shifting: On the Relationship between Personal and Corporate Tax Rates, in: Ifo-Studien 48: 4, 611-632.

Ganghof, Steffen 1999: Steuerwettbewerb und Vetospieler. Stimmt die These der blockierten Anpassung?, in: Politische Vierteljahresschrift 40: 3, 458-472.

Ganghof, Steffen 2000: Adjusting National Tax Policy to Economic Internationalization, in: Scharpf, Fritz W./Schmidt, Vivien A. (Hrsg.): Welfare and Work in the Open Economy, Band 2: Diverse Responses to Common Challenges, Oxford, 597-645.

Ganghof, Steffen 2003: Promises and Pitfalls of Veto Player Analysis, in: Swiss Political Science Review 9: 2, 1-25.

Ganghof, Steffen 2004: Wer regiert in der Steuerpolitik? Einkommensteuerreform zwischen internationalem Wettbewerb und nationalen Verteilungskonflikten, Frankfurt a. M.

Ganghof, Steffen 2005: The Politics of Income Taxation: A Comparative Analysis, Colchester, i. E.

Ganghof, Steffen/Bräuninger, Thomas 2005: Government Status and Legislative Behavior: Partisan Veto Players in Australia, Denmark, Finland and Germany, in: Party Politics, i. E.

Ganghof, Steffen/Eccleston, Richard 2004: Globalisation and the Dilemmas of Income Taxation in Australia, in: Australian Journal of Political Science 39: 4, 519-534.

Garrett, Geoffrey 1998a: Partisan Politics in the Global Economy, Cambridge. 
Garrett, Geoffrey 1998b: Shrinkinig States? Globalization and National Autonomy in the OECD, in: Oxford Development Studies 26: 1, 71-97.

Genschel, Philipp 2003: Globalisierung als Problem, als Lösung und als Staffage, in: Hellmann, Gunther/Wolf, Klaus Dieter/Zürn, Michael (Hrsg.): Die neuen Internationalen Beziehungen. Forschungsstand und Perspektiven in Deutschland, Baden-Baden, 429464.

Goldstein, Judith/Keohane, Robert O. 1993: Ideas and Foreign Policy: An Analytical Framework, in: Goldstein, Judith/Keohane, Robert O. (Hrsg.): Ideas and Foreign Policy: Beliefs, Institutions, and Political Change, Ithaca, NY, 3-30.

Grubert, Harry 2001: Tax Planning by Companies and Tax Competition by Governments: Is There Evidence of Changes of Behavior?, in: Hines, James R. (Hrsg.): International Taxation and Multinational Activity, Chicago, IL, 113-139.

Hall, Peter A. 1993: Policy Paradigms, Social Learning, and the State: The Case of Economic Policymaking in Britain, in: Comparative Politics 25: 3, 275-296.

Hallerberg, Mark/Basinger, Scott 1998: Internationalization and Changes in Tax Policy in OECD Countries: The Importance of Domestic Veto Players, in: Comparative Political Studies 31: 3, 321-352.

Haufler, Andreas/Schjelderup, Guttorm 2000: Corporate Tax Systems and Cross Country Profit Shifting, in: Oxford Economic Letters 52: 2, 306-325.

Hines Jr., James R. 1999: Lessons form Behavioral Responses to International Taxation, in: National Tax Journal 52: 2, 305-322.

Hobson, John M. 2003: Disappearing Taxes or the »Race to the Middle«? Fiscal Policy in the OECD, in: Weiss, Linda (Hrsg.): States in the Global Economy, Sydney, 37-57.

Homburg, Stefan 2000: Allgemeine Steuerlehre, 2. Auflage, München.

Huber, Evelyne/Ragin, Charles/Stephens, John D. 1993: Social Democracy, Christian Democracy, Conditional Structure and the Welfare State, in: American Journal of Sociology 99: 3, 711-749.

Immergut, Ellen 1992: Health Politics: Interests and Institutions in Western Europe, Cambridge.

Isaac, John 1997: A Comment on the Viability of the Allowance for Corporate Equity, in: Fiscal Studies 18: 3, 303-318.

Jachtenfuchs, Markus 1995: Ideen und internationale Beziehungen, in: Zeitschrift für Internationale Beziehungen 2: 2, 417-442.

Keen, Michael/King, John 2003: The Croatian Profit Tax: An ACE in Practice, in: Rose, Manfred (Hrsg.): Integriertes Steuer- und Sozialsystem, Heidelberg, 323-342.

King, Gary 1991: »Truth « is Stranger than Prediction, More Questionable than Causal Inference, in: American Journal of Political Science 35: 4, 1047-1053.

Lee, Dwight/McKenzie, Richard 1989: The International Political Economy of Declining Tax Rates, in: National Tax Journal 42: 1, 79-83.

Lijphart, Arend 1999: Patterns of Democracy, New Haven, CT.

Maier, Matthias L. 2003: Wissens- und ideenorientierte Ansätze in der Politikwissenschaft. Versuch einer systematischen Übersicht, in: Maier, Matthias L./Hurrelmann, Achim/ Nullmeier, Frank/Pritzlaff, Tanja/Wiesner, Achim (Hrsg.): Politik als Lernprozess. Wissenszentrierte Ansätze der Politikanalyse, Opladen, 25-77.

Martin, Christian W. 2004: Die doppelte Transformation. Demokratie und Außenwirtschaftsliberalisierung in Entwicklungsländern, Opladen.

Messere, Ken 1997: OECD Tax Developments in the 1990s, in: Bulletin for International Fiscal Documentation 51: 7, 298-314.

Mintz, Jack M. 1995: Corporation Tax: A Survey, in: Fiscal Studies 16: 4, 23-68.

Mulé, Rosa 2001: Political Parties, Games and Redistribution, Cambridge.

Musgrave, Richard A. 2002: Equity and the Case for Progressive Taxation, in: Thorndike, Joseph J./Ventry Jr., Dennis J. (Hrsg.): Tax Justice: The Ongoing Debate, Washington, DC, 9-24.

Nagel, Jack H. 1998: Social Choice in a Pluralitarian Democracy: The Politics of Market Liberalization in New Zealand, in: British Journal of Political Science 28: 2, 223-267. 
Office of Tax Policy Research 2004: World Tax Database, in: http://www.bus.umich.edu/ OTPR/otpr/introduction.htm; 11.1.2004.

Ottaviani, Marco 2002: Comment, in: Economic Policy 17: 35, 490-492.

Plümper, Thomas/Schulze, Günther G. 1999: Steuerwettbewerb und Steuerreformen, in: Politische Vierteljahresschrift 40: 3, 445-457.

Quah, Dennis 1993: Galton's Fallacy and Tests of the Convergence Hypothesis, in: Scandinavian Journal of Economics 95: 4, 427-443.

Sala-i-Martin, Xavier X. 1996: The Classical Approach to Convergence Analysis, in: The Economic Journal 106: 2, 1019-1036.

Salsbäck, Johan 1993: The Tax Reform Process in Sweden, in: Nordic Council for Tax Research (Hrsg.): Tax Reform in the Nordic Countries, Uppsala, 199-212.

Slemrod, Joel 2004: Are Corporate Tax Rates, or Countries, Converging?, in: Journal of Public Economics 88: 6, 1169-1186.

Sørensen, Peter Birch 2001: The Nordic Dual Income Tax: In or Out?, unv. Ms.

Steinmo, Sven 2003: The Evolution of Policy Ideas: Tax Policy in the 20th Century, in: British Journal of Politics and International Relations 5: 2, 206-236.

Swank, Duane 1998: Funding the Welfare State: Globalization and the Taxation of Business in Advanced Market Economies, in: Political Studies 46: 4, 671-692.

Swank, Duane 2003: Tax Policy in an Era of Internationalization: An Assessment of a Conditional Diffusion Model of the Spread of Neoliberalism, unv. Ms.

Swank, Duane/Steinmo, Sven 2002: The New Political Economy of Taxation in Advanced Capitalist Democracies, in: American Journal of Political Science 46: 3, 642-655.

Tsebelis, George 2002: Veto Players: How Political Institutions Work, Princeton, NJ.

Wagschal, Uwe 1999a: Blockieren Vetospieler Steuerreformen?, in: Politische Vierteljahresschrift 40: 4, 628-640.

Wagschal, Uwe 1999b: Schranken staatlicher Steuerungspolitik. Warum Steuerreformen scheitern können, in: Busch, Andreas/Plümper, Thomas (Hrsg.): Nationaler Staat und internationale Wirtschaft, Baden-Baden, 223-247.

Wagschal, Uwe 2001: Deutschlands Steuerstaat und die vier Welten der Besteuerung, in: Schmidt, Manfred G. (Hrsg.): Wohlfahrtsstaatliche Politik: Institutionen - Prozesse Leistungsprofil, Opladen, 126-162.

Wala, Thomas 1999: Der Abzug fiktiver Eigenkapitalzuwachszinsen als »Sparvariante « einer zinsbereinigten Gewinnsteuer. Was bringt der neue § 111 EStG?, in: RWZ Zeitschrift für Recht und Rechnungswesen (1999): 6, 166-170.

Wallerstein, Michael/Przeworski, Adam 1995: Capital Taxation with Open Borders, in: Review of International Political Economy 2: 3, 425-445.

Wilson, John D. 1991: Tax Competition with Interregional Differences in Factor Endowments, in: Regional Science and Urban Economics 21: 3, 423-451. 